\title{
Opposing actions of angiopoietin-2 on Tie2 signaling and FOXO1 activation
}

\author{
Minah Kim, ${ }^{1}$ Breanna Allen, ${ }^{1}$ Emilia A. Korhonen, ${ }^{2}$ Maximilian Nitschké, ${ }^{1}$ Hee Won Yang, ${ }^{3}$ Peter Baluk, ${ }^{1}$ \\ Pipsa Saharinen, ${ }^{2}$ Kari Alitalo, ${ }^{2}$ Christopher Daly, ${ }^{4}$ Gavin Thurston, ${ }^{4}$ and Donald M. McDonald ${ }^{1}$ \\ 'Department of Anatomy, Cardiovascular Research Institute, and UCSF Helen Diller Family Comprehensive Cancer Center, UCSF, San Francisco, California, USA. Wihuri Research Institute, \\ Translational Cancer Biology Program, University of Helsinki, Helsinki, Finland. ${ }^{3}$ Department of Chemical and Systems Biology, Stanford University School of Medicine, \\ Stanford, California, USA. ${ }^{4}$ Regeneron Pharmaceuticals Inc., Tarrytown, New York, USA.
}

\begin{abstract}
Angiopoietin-2 (ANG2) regulates blood vessel remodeling in many pathological conditions through differential effects on Tie2 signaling. While ANG2 competes with ANG1 to inhibit Tie2, it can paradoxically also promote Tie2 phosphorylation ( $p$-Tie2). A related paradox is that both inactivation and overactivation of Tie2 can result in vascular remodeling. Here, we reconciled these opposing actions of ANG2 by manipulating conditions that govern its actions in the vasculature. ANG2 drove vascular remodeling during Mycoplasma pulmonis infection by acting as a Tie2 antagonist, which led to $\mathrm{p}$-Tie2 suppression, forkhead box 01 (FOX01) activation, increased ANG2 expression, and vessel leakiness. These changes were exaggerated by anti-Tie2 antibody, inhibition of PI3K signaling, or ANG2 overexpression and were reduced by anti-ANG2 antibody or exogenous ANG1. In contrast, under pathogen-free conditions, ANG2 drove vascular remodeling by acting as an agonist, promoting high p-Tie2, low FOXO1 activation, and no leakage. Tie1 activation was strong under pathogen-free conditions, but infection or TNF- $\alpha$ led to Tie1 inactivation by ectodomain cleavage and promoted the Tie2 antagonist action of ANG2. Together, these data indicate that ANG2 activation of Tie2 supports stable enlargement of normal nonleaky vessels, but reduction of Tie1 in inflammation leads to ANG2 antagonism of Tie2 and initiates a positive feedback loop wherein FOXO1-driven ANG2 expression promotes vascular remodeling and leakage.
\end{abstract}

\section{Introduction}

Vascular remodeling, resulting from molecular, structural, and functional changes in endothelial cells of the microcirculation, contributes to plasma leakage and leukocyte influx in inflammation and other conditions. The remodeling is initiated by multiple cytokines, including VEGF, TNF- $\alpha$, and the angiopoietins, angiopoietin-1 (ANG1, also known as ANGPT1) and ANG2 (also known as ANGPT2) (1-4).

Angiopoietins are vascular regulators that act on Tie receptors, Tie1 and Tie2 (also known as Tek), under both normal and pathologic conditions. ANG1 is a constitutive agonist for Tie2 phosphorylation ( $\mathrm{p}$-Tie2) that contributes to vascular remodeling during development and maintenance of vascular stability thereafter (5-7). Deletion of either Ang1 or Tie2 results in embryonic lethality (6). No ligand has been reported for Tie1, but Tie1 deletion during development is also lethal $(5,8)$. The interaction of Tie1 and Tie2 can modulate angiopoietin-induced Tie2 phosphorylation (9). ANG1 activation of Tie2 promotes vascular maturation and stabilization through Akt, which phosphorylates forkhead box O1 (FOXO1 ) transcription factor (10-14). Phos-

\section{Related Commentary: p. 3188}

Conflict of interest: C. Daly and C. Thurston are employees of Regeneron Pharmaceuticals Inc.

Submitted: September 30, 2015; Accepted: June 24, 2016.

Reference information: J Clin Invest. 2016;126(9):3511-3525. doi:10.1172/JCI84871. phorylation of FOXO1 inhibits its transcriptional function by promoting nuclear exclusion and preventing DNA binding $(10,14)$. ANG1-mediated inhibition of Foxo1 transcriptional activation in endothelial cells induces expression of genes involved in vessel stability and repression of ANG2 and other genes involved in vascular destabilization $(11,13)$.

ANG2 competitively inhibits the action of ANG1 on Tie2 (7, 15) and promotes vascular remodeling by suppression of Tie2 signaling (4, 16-19). ANG2 overexpression causes embryonic lethality similar to deletion of Ang1 or Tie2 (15). A seemingly paradoxical but well-documented action of ANG2 is the activation of Tie 2 under certain conditions $(15,20,21)$. ANG2 acts as a weak agonist of Tie2 when used in high concentrations, in the absence of ANG1, or on certain cell types $(15,20,22,23)$. In otherwise normal mice, ANG2 can activate Tie2/Akt signaling and FOXO1 phosphorylation, suppress ANG2 production, and decrease plasma leakage (20). The mechanism underlying the context-dependent change of ANG2 function from Tie2 agonist to antagonist is unknown.

Tie2 signaling is controlled by the balance of agonistic actions of ANG1 and antagonistic or agonistic actions of ANG2. ANG1 dominates in quiescent blood vessels, but ANG2 plays important roles in inflammation, sepsis, and other pathological conditions $(18,24-26)$ and is rapidly released from endothelial cells by inflammatory stimuli $(27,28)$. The effects of ANG2 are greatly amplified by TNF- $\alpha$ and other inflammatory cytokines $(4,16,17$, 27). ANG2 inhibitors can reduce vascular remodeling and inflam- 
A

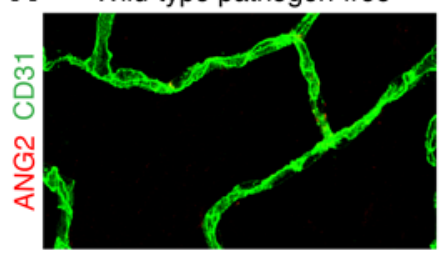

B Ang2-EGFP pathogen-free

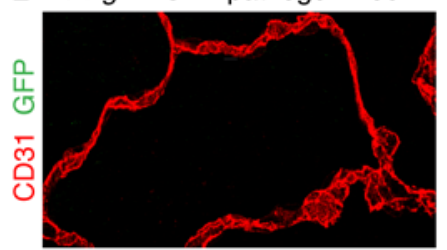

C

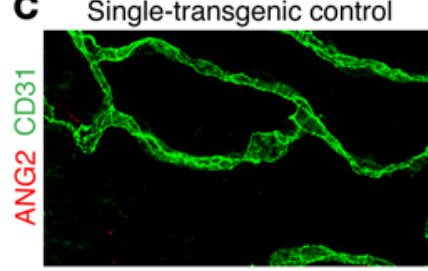

Dild-type control

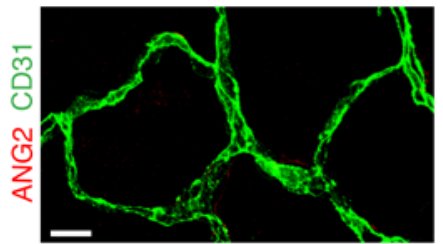

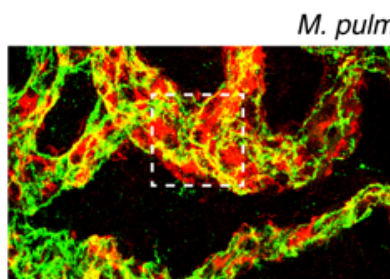

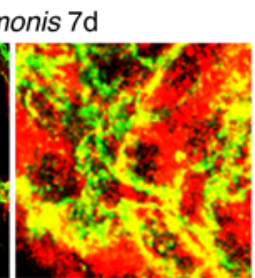

M. pulmonis $7 \mathrm{~d}$

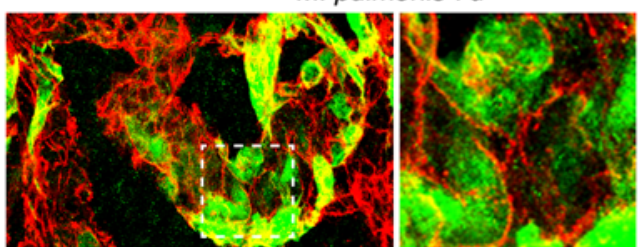

Tie1-ANG2
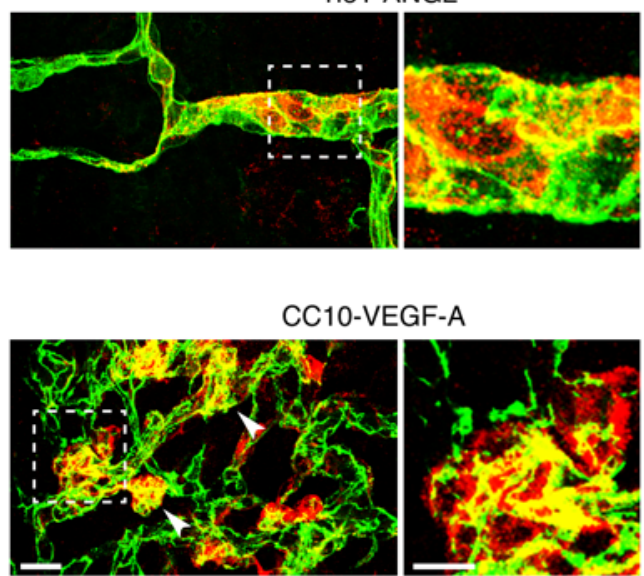

$\mathbf{E}$

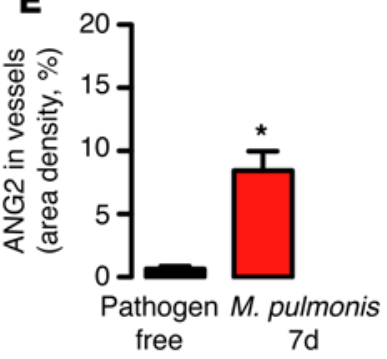

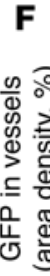

G
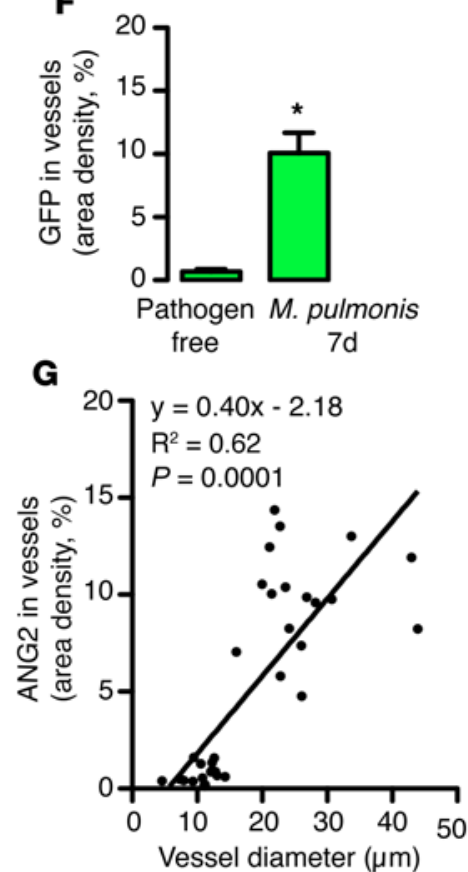

Figure 1. ANG2 expression in endothelial cells at sites of vascular remodeling. ( $\mathbf{A}$ and $\mathbf{B}$ ) Weak or absent ANG2 immunofluorescence in normal tracheal blood vessels of pathogen-free wild-type mouse (A) and Ang2-EGFP mouse (B) compared with strong ANG2 staining in remodeled vessels in both types of mice after M. pulmonis infection for 7 days. (C) Comparison of no ANG2 staining in vessels of control mouse with strong staining in enlarged vessels after ANG2 overexpression in endothelial cells of Tie1-ANG2 mouse off doxycycline from birth to 8 weeks. (D) Comparison of no ANG2 staining in vessels of control mouse with strong staining in bulbous vascular expansions (arrowheads) after VEGF-A overexpression in CC10-VECF-A mouse on doxycycline for 7 days. Boxed region enlarged on the right. Scale bars: $10 \mu \mathrm{m}$. (E and F) Comparison of ANG2 immunofluorescence of tracheal blood vessels in C57BL/6 mice (E) and EGFP staining in Ang2-EGFP mice (F) under pathogen-free conditions and after infection for 7 days ( $n=12$ per group). ${ }^{*} P<0.05$ vs. pathogen-free, Student's $t$ test. (G) Correlation $(P=0.0001)$ between amount of ANG2 staining and size of 34 tracheal vessels in 12 pathogen-free Tie1-ANG2 mice off doxycycline from birth to 8 weeks of age.

mation severity $(4,18)$ and are more effective when combined with an inhibitor of TNF- $\alpha$ (17). The role of Tie1 in these events is unclear, but TNF- $\alpha$ can trigger Tie1 inactivation by shedding of the extracellular domain (29).

Another paradoxical feature of angiopoietins is that vascular remodeling is not only driven by Tie 2 inactivation, but also by Tie 2 overactivation (20,30-32). Activating mutations in the Tie2 gene, transgenic overexpression, or administration of exogenous ANG1 or ANG2 alone can all induce vascular enlargement (30-34). The enlarged vessels have superficial similarities to leak-prone venules, but are resistant to leakage induced by inflammatory mediators $(31,32,35-37)$.

In the present study, we examine these paradoxes and describe a potential mechanism of the context-dependent action of ANG2 by manipulating its interaction with Tie2 in the respiratory tract under normal conditions and in inflammation. We first asked whether ANG2 protein expression changed in synchro- ny with vascular remodeling by using transgenic reporter mice (Ang2-EGFP) and mice that overexpress ANG2 (Tie1-ANG2 mice) $(38,39)$ or VEGF (CC10-VEGF-A mice, where CC10 indicates club cell $10 \mathrm{kDa}$ ) (40). Mycoplasma pulmonis infection was used as a model of vascular remodeling in inflammation (4). Gain- and loss-of-function strategies were used with structure/function readouts to determine how ANG2 regulation of Tie2 was mechanistically implicated in vascular remodeling. We then identified a link between Tie 2 activity and ANG2 expression by elucidating the temporal sequence of changes in Tie2, FOXO1 transcriptional activity, and ANG2 expression in endothelial cells in vivo. The mechanism determining whether ANG2 acted as a Tie2 agonist or antagonist was further examined by comparing changes in Tie1 and Tie2 during infection or TNF- $\alpha$ exposure.

We found that ANG2 agonism of Tie2 promoted stable enlargement of normal vessels. However, in inflammation, loss of Tie1 by ectodomain shedding promoted the antagonistic 
A M. pulmonis

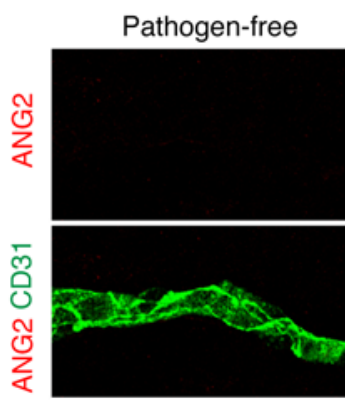

B

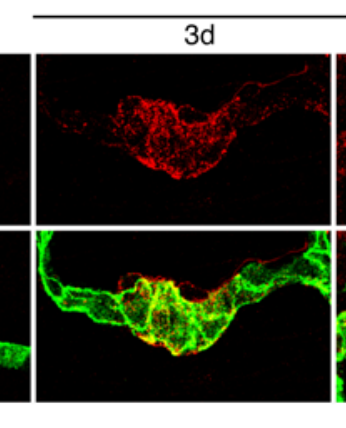

M. pulmonis

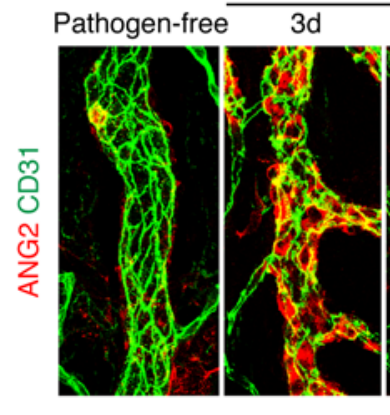

D
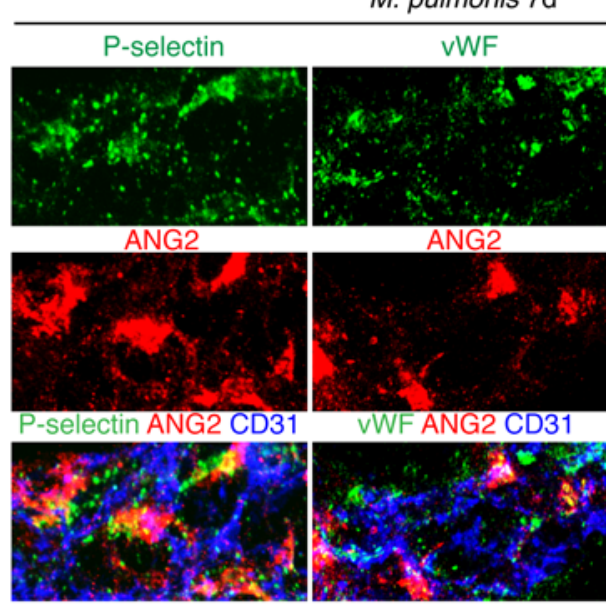

M. pulmonis 7d
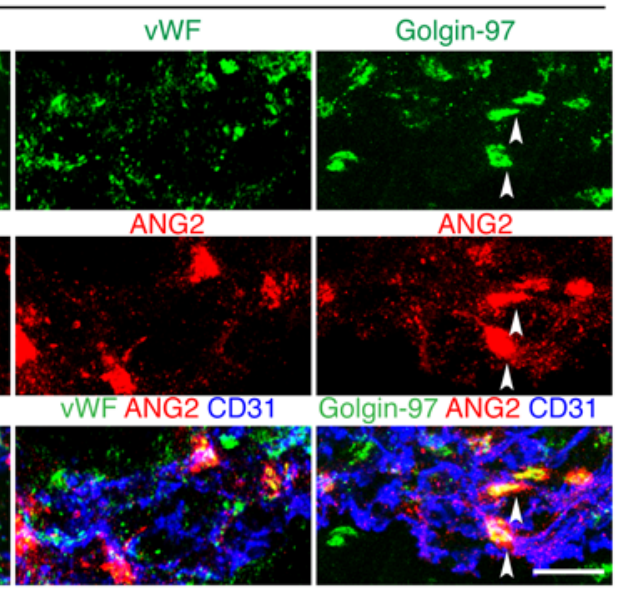

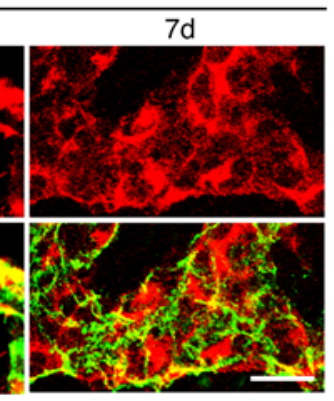

c
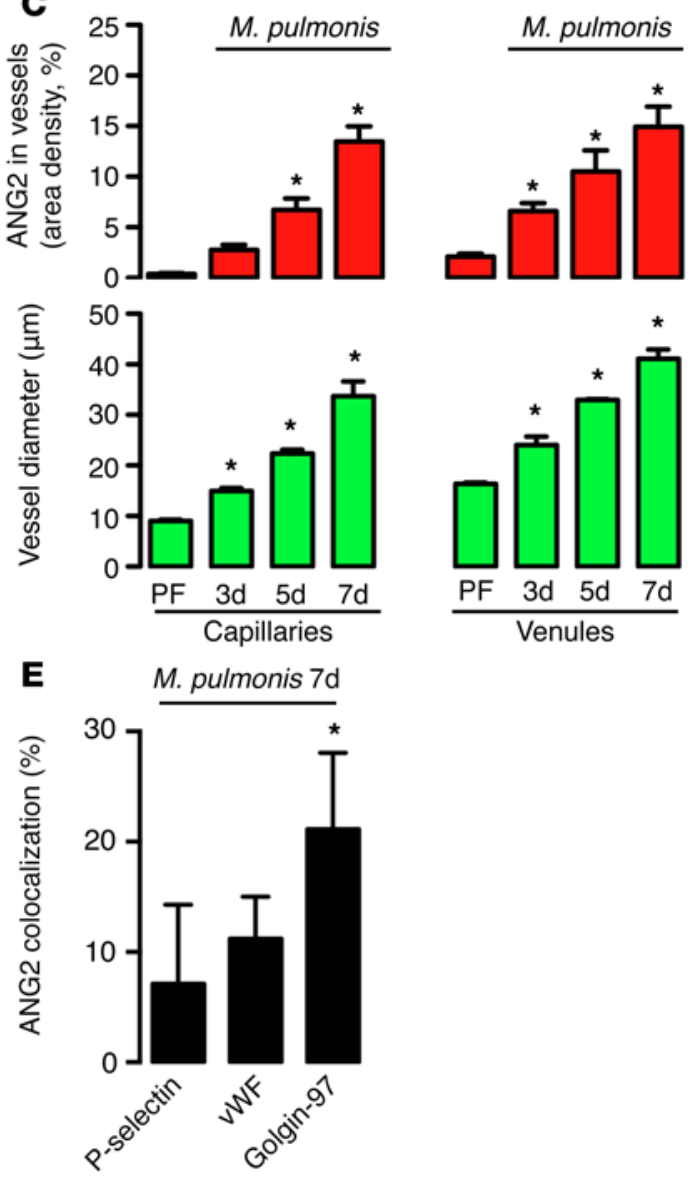

Figure 2. Temporal change in ANG2 expression and subcellular distribution during vascular remodeling after infection. (A) Progressive increase in ANG2 immunofluorescence at sites of capillary remodeling over cartilage rings at days 3, 5, and 7 after M. pulmonis infection in C3H mice. ANG2 staining in enlarged vessel is strong at 3 days and even stronger and more uniform at 7 days after infection. (B) More rapid increase in ANG2 at sites of venule remodeling between cartilage rings at 3 days after infection. ANG2 staining is uniformly strong in venules at days 3, 5 , and 7 of infection. Scale bars: $25 \mu \mathrm{m}$ (A and B). (C) Parallel increases in amount of ANC2 staining (upper) and size of capillaries and venules (lower) after infection ( $n=9$ per group). ${ }^{*} P<0.05$ vs. pathogen-free, 1-way ANOVA. (D) Strong colocalization of ANG2 with golgin-97 and weak colocalization with P-selectin and vWF in blood vessels at 7 days after infection in C57BL/6 mice. Arrowheads mark sites of ANG2 colocalization with golgin-97. Scale bar: $10 \mu \mathrm{m}$. (E) Significantly greater colocalization of ANG2 with golgin-97 than with P-selectin or VWF ( $n=9$ per group). ${ }^{*} P<0.05$ vs. P-selectin or VWF, 1-way ANOVA.

action of ANG2 on Tie2 and a positive feedback loop, whereby $\mathrm{p}$-Tie2 suppression, FOXO1 transcriptional activation, and increased ANG2 expression led to vascular remodeling.

\section{Results}

ANG2 expression in endothelial cells at sites of vascular remodeling. To determine whether ANG2 expression in endothelial cells increases at sites of vascular remodeling, we compared the amount of
ANG2 protein in the tracheal vasculature under different states of growth and remodeling. To visualize ANG2, we used a human monoclonal antibody (REGN910) that has high binding affinity to murine ANG2 $\left(K_{D}=0.09 \mathrm{nM}\right)$ and does not bind ANG1 of either species (24). In tests of validation, we found that REGN910 recognized ANG2 in ovarian follicles and in vessels of ovarian cortex and endometrium, known locations of expression (15, 27). ANG2 in cultured endothelial cells was also stained by REGN910 under 
A

Pathogen-free
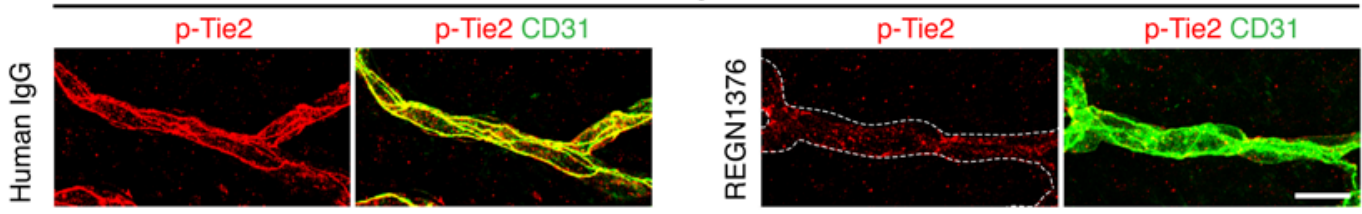

B

M. pulmonis $7 \mathrm{~d}$
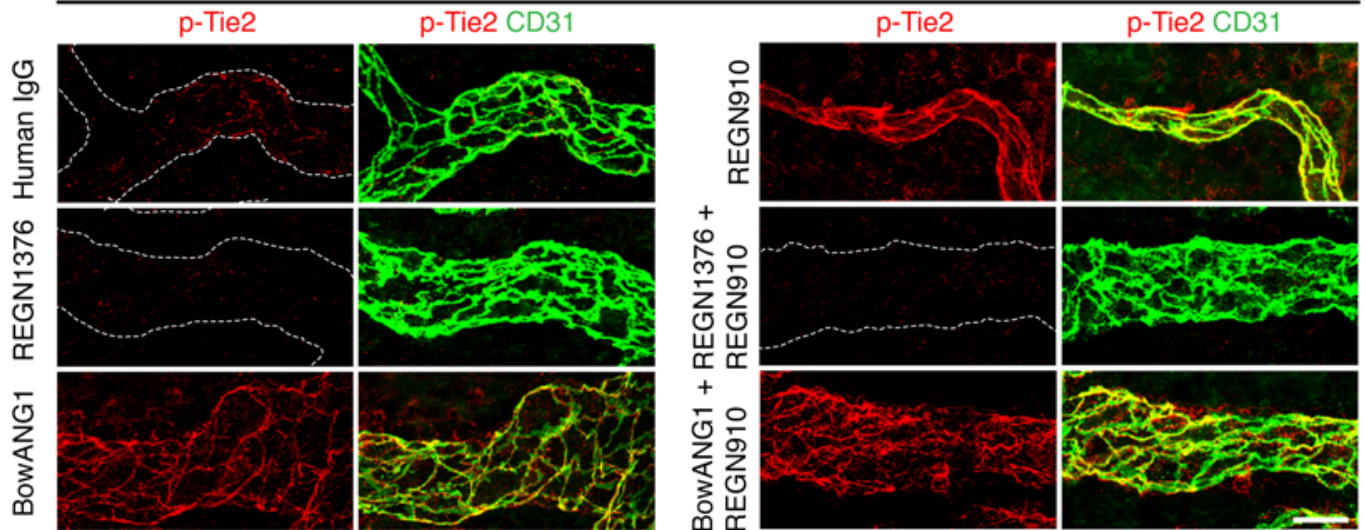

C

Pathogen-free

M. pulmonis 7d

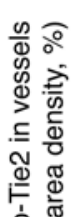

157

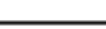

๓๐ Щ
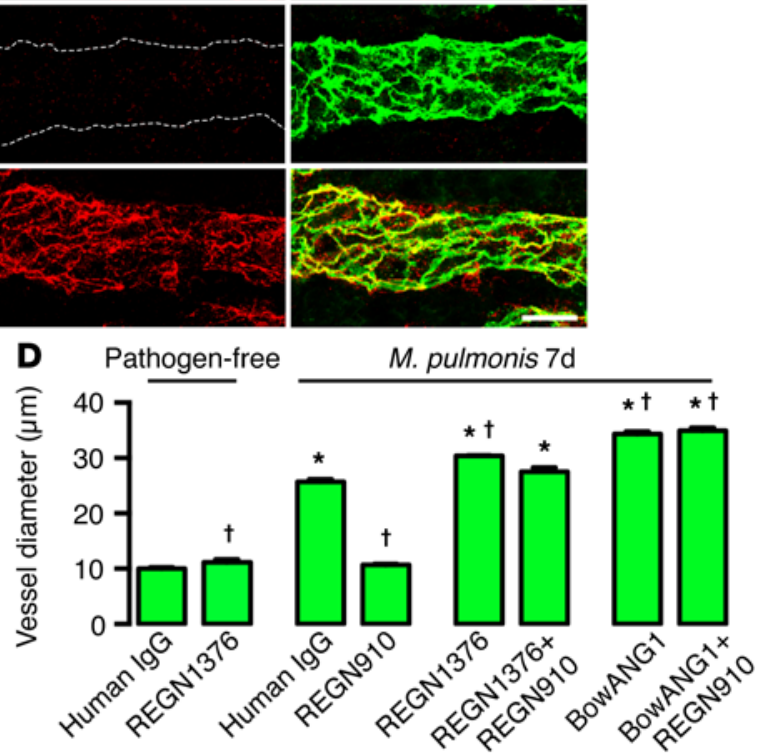

E
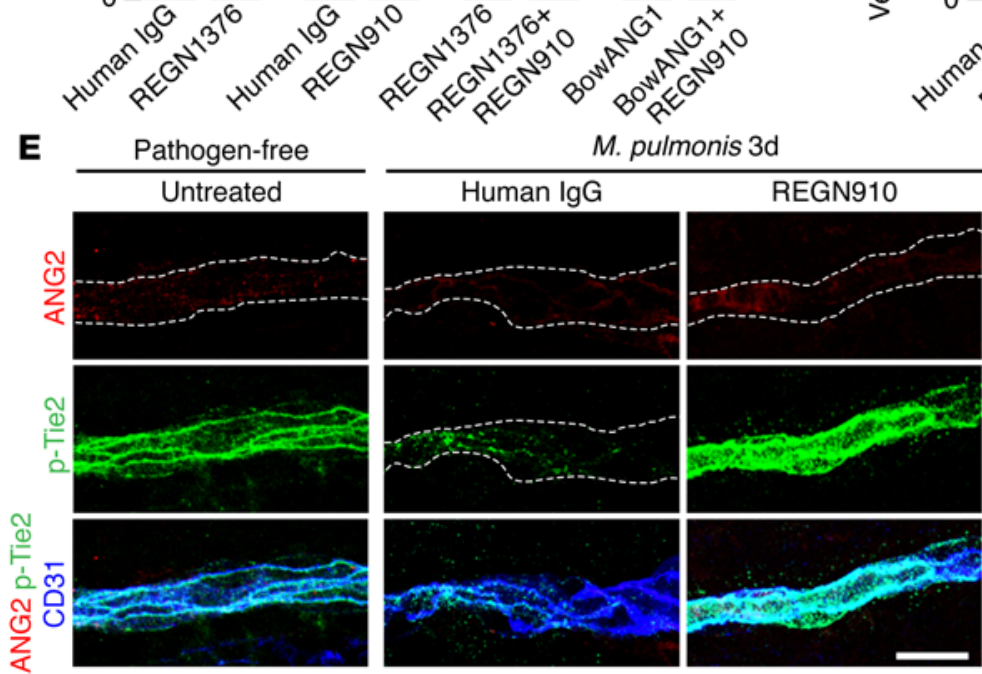

F

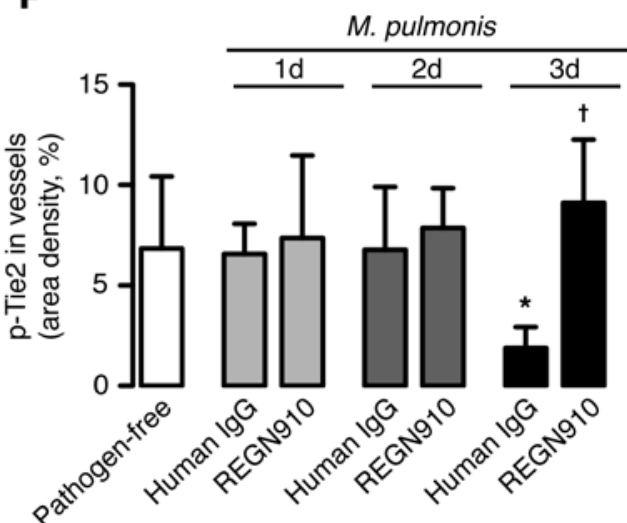

Figure 3. ANG2 effects on Tie2 phosphorylation and vascular enlargement. (A) Blood vessels of pathogen-free mice comparing strong $p$-Tie2 staining in a control (human IgG) with weak p-Tie2 after inhibition of Tie2 by REGN1376. (B) Blood vessels after M. pulmonis infection for 7 days showing weak $p$-Tie2 in remodeled vessels in a control (human IgG) compared with strong p-Tie2 after inhibition of total2 by REGN910. Weak or absent p-Tie2 after REGN1376 alone or with REGN910, but stronger p-Tie2 after BowANG1 or BowANG1 plus REGN910, indicating that BowANG1 overcame ANG2 suppression of Tie2 after infection. ANG2 inhibition also reduced vascular remodeling, but Tie2 inhibition exaggerated the enlargement and blocked the effect of REGN910. BowANG1 alone or together with REGN910 amplified the enlargement. Dashed white lines delineate vessels stained by CD31. (C and D) Measurements of $\mathrm{p}$-Tie2 staining (C) and vessel size (D), comparing groups shown in $\mathbf{A}$ and $\mathbf{B}\left(n=12\right.$ per group). ${ }^{*} P<0.05$ vs. pathogen-free lgG controls; ${ }^{\dagger} P<0.05$ vs. infected IgG controls, 1-way ANOVA. (E) Comparison of ANG2 and p-Tie2 at baseline after 3 days of infection, showing that $p$-Tie2 reduction after infection was blocked by ANG2 inhibition. ANG2 staining was weak in all groups at this time point. Scale bars: $20 \mu \mathrm{m}$. (F) Measurements showing that the reduction in $\mathrm{p}$-Tie2 staining after infection was not evident until 3 days and that this reduction was blocked by inhibition of ANG2 ( $n=6$ per group). ${ }^{*} P<0.05$ vs. pathogen-free control; ${ }^{\dagger} P<0.05$ vs. infected IgG controls at 3 days, 1-way ANOVA. 
A

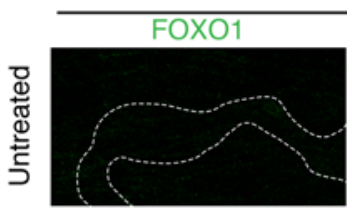

B

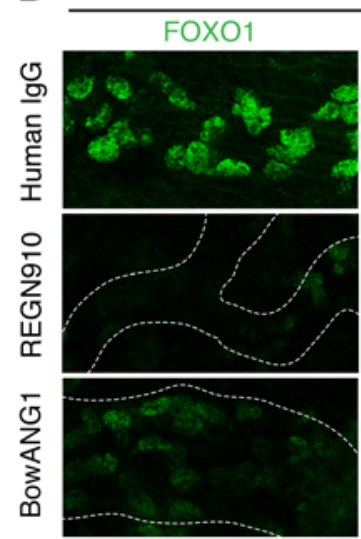

D

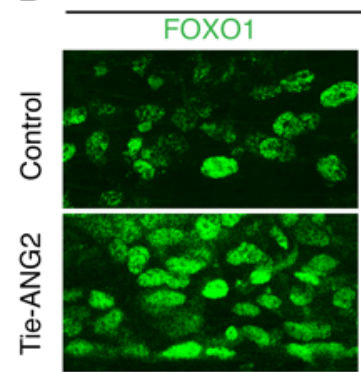

G $\frac{\text { Human IgG, M. pulmonis 7d }}{\text { ANG2 fluorescence intensity }}$ Control

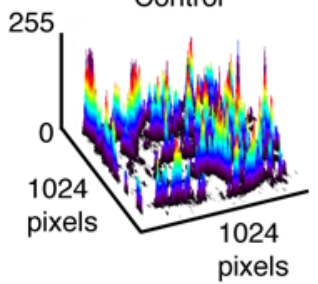

Pathogen-free

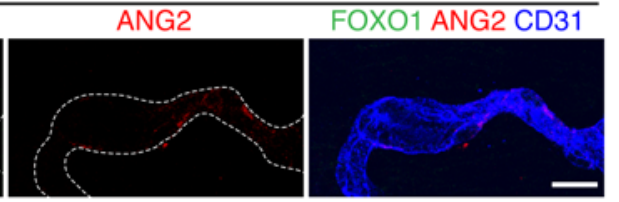

M. pulmonis 7d

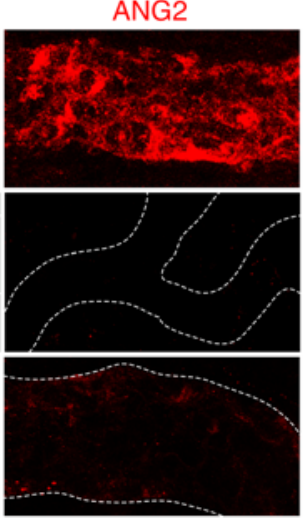

M. pulmonis 7d

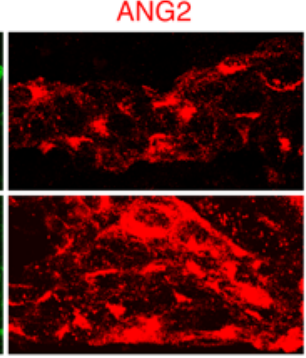

REGN910, M. pulmonis 7d

ANG2 fluorescence intensity Tie1-ANG2

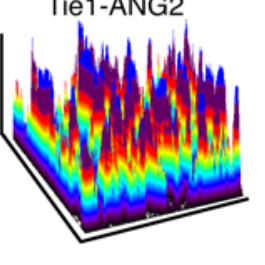

FOXO1 ANG2 CD31

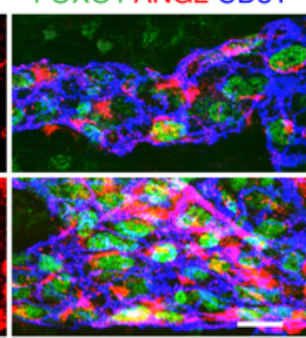
Control

FOXO1 ANG2 CD31
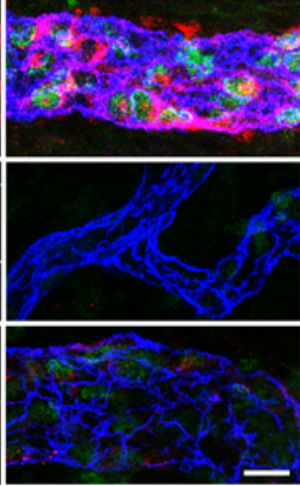

255

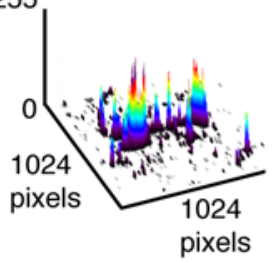

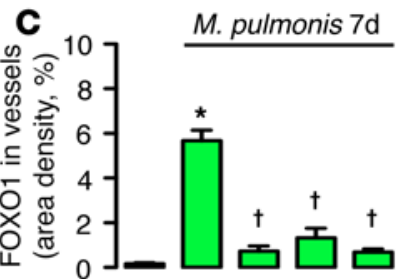

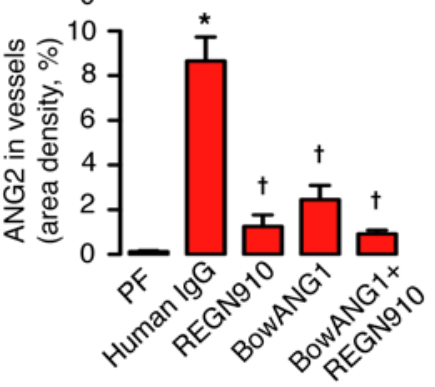

F
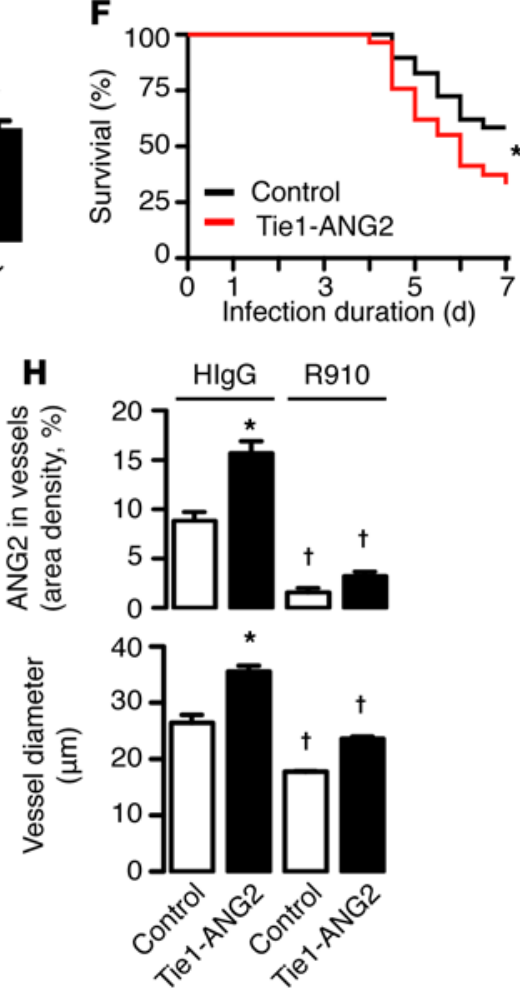

Figure 4. ANG2-dependent parallel increase in F0X01 activation and ANG2 expression after infection. (A) Weak or absent F0X01 and ANG2 immunofluorescence in normal vessel of pathogen-free wild-type mouse. (B) Blood vessels of mice with M. pulmonis infection for 7 days, showing strong nuclear FOXO1 staining and cytoplasmic ANG2 in a remodeled vessel after control treatment (human IgG). FOXO1 and ANG2 are weak after ANG2 blockade by REGN910 or after Tie2 overactivation by BowANG1 alone or with REGN910. Dashed white lines delineate vessel borders marked by CD31. (C) Measurements of FOX01 (top) and ANG2 (bottom) staining in groups shown in $\mathbf{A}$ and $\mathbf{B}$ ( $n=12$ per group). ${ }^{*} P<0.05$ vs. pathogen-free controls; ${ }^{\dagger} P<0.05$ vs. infected lgG controls, 1 -way ANOVA. (D) Comparison of control and Tie1-ANG2 mice infected for 7 days showing strong FOX01 and ANG2 immunoreactivity, exaggerated in Tie1-ANG2 mouse. (E) Measurements comparing amounts of FOX01 staining in infected control and Tie1-ANG2 mice ( $n=6$ per group). ${ }^{*} P<0.05$, Student's $t$ test. (F) Kaplan-Meier survival curves for control (black) and Tie1-ANG2 (red) mice over 7 days of infection ( $n=21$ per group). ${ }^{*} P<0.05$, log-rank test. (G) Surface plots of confocal microscopic images showing intensity of ANG immunofluorescence in control and Tie1-ANG2 mice infected for 7 days and treated with control antibody (human IgG) or anti-ANG2 (REGN910). Increase in ANG2 immunofluorescence and vascular enlargement after infection was exaggerated in Tie1ANG2 mice, blocked by REGN910. Scale bars: $20 \mu \mathrm{m}$. (H) Measurements showing amounts of ANG2 immunofluorescence (upper) and vessel size (lower) in groups shown in $\mathbf{G}\left(n=12\right.$ per group). ${ }^{*} P<0.05$ vs. corresponding control group; ${ }^{\dagger} P<0.05$ vs. corresponding IgG-treated group, 1 -way ANOVA.

baseline conditions, where most colocalized with vWF, but not after phorbol diester treatment, which is known to evoke ANG2 release (28) (Supplemental Figure 1, A-C; supplemental material available online with this article; doi:10.1172/JCI84871DS1).
ANG2 immunoreactivity was weak or absent in tracheal blood vessels of pathogen-free C57BL/6 mice, but was moderate to strong in enlarged vessels after M. pulmonis infection for 7 days (Figure 1, $\mathrm{A}$ and $\mathrm{E}$ ), when the vasculature is known to be extensively remod- 
A

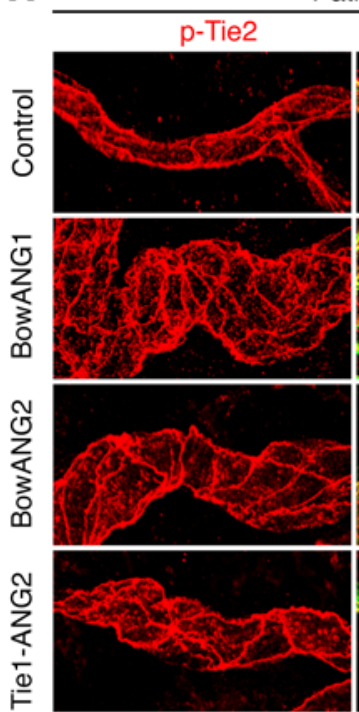

Pathogen-free
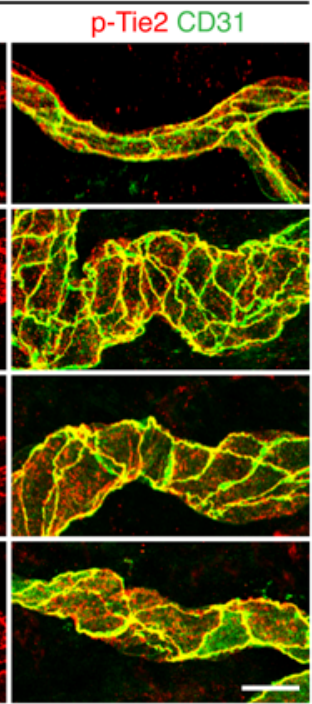

C

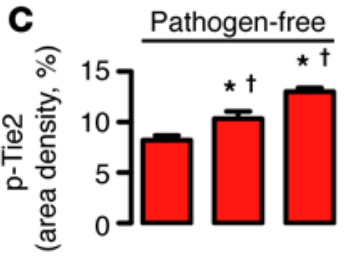

M. pulmonis 7d
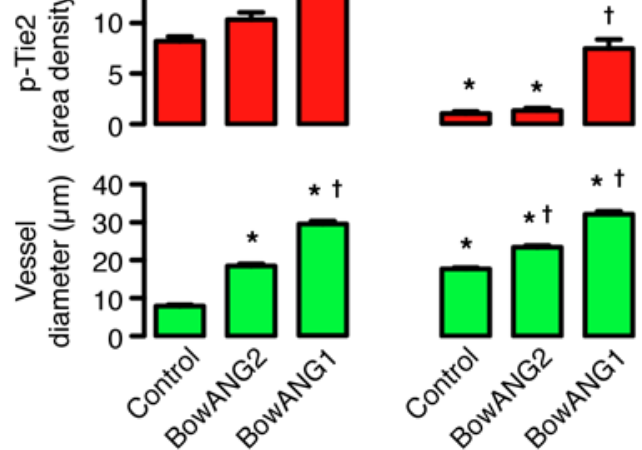

$\mathbf{B}$

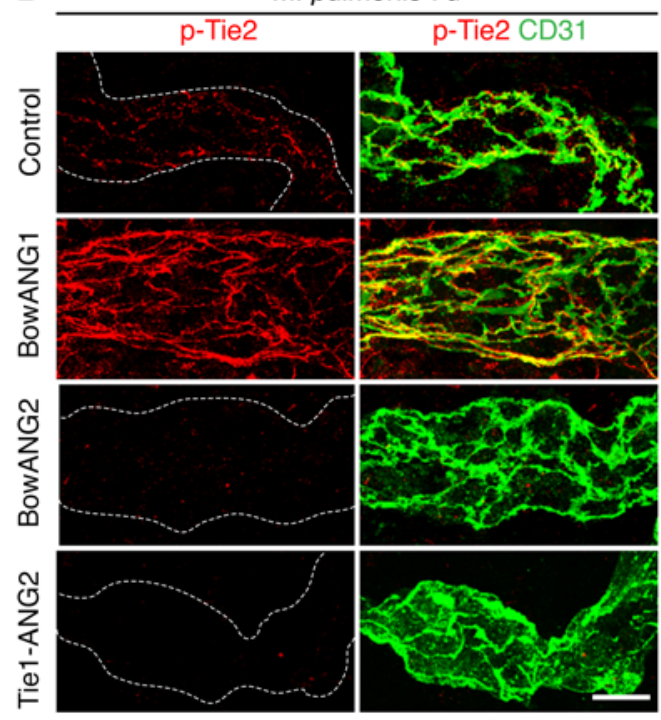

D Pathogen-free M. pulmonis 7d
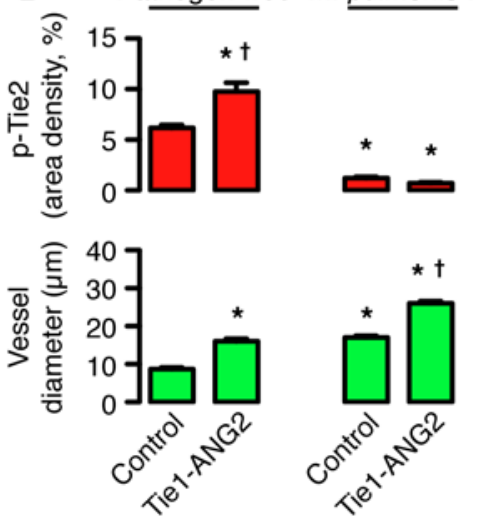

Figure 5. Contrasting actions of ANG2 with or without infection. (A) Strong p-Tie2 immunofluorescence in pathogen-free control mouse, wild-type mouse given BowANG1 or BowANG2 for 7 days, and Tie1-ANG2 mouse aged 8 weeks. (B) Comparison of the same 4 groups after 7 days of M. pulmonis infection, where p-Tie2 staining is much weaker in all mice except the one treated with BowANG1. Dashed white lines delineate vessel borders marked by CD31. Scale bars: $20 \mu \mathrm{m}$. (C and D) Measurement of p-Tie2 immunofluorescence (upper) and vessel size (lower) in pathogen-free or infected wild-type mice given BowANG1 or BowANG2 for 7 days (C) and Tie1-ANG2 mice aged 8 weeks (D) compared with respective controls ( $n=4-5$ per group). ${ }^{*} P<0.05$ vs. pathogen-free controls; ${ }^{\dagger} P<0.05$ vs. infected controls, 1-way ANOVA.

eled $(4,41,42)$. As another approach to evaluating the significance of strong ANG2 staining in remodeled vessels, we used Ang2-EGFP transgenic reporter mice, prepared by using BAC technology (43) (Supplemental Figure 2, A and B). ANG2 expression reflected by EGFP immunoreactivity was similarly strong at sites of vascular remodeling in these mice at 7 days after infection, but was faint or absent under normal conditions (Figure 1, B and F). Specificity for ANG2 in these mice was confirmed by the presence of strong EGFP staining in hippocampal neurons (Supplemental Figure 2C), as reported in GENSAT (http://www.gensat.org/).

ANG2 staining was also strong in focal regions of vascular enlargement in the trachea of conditional Tie1-ANG2-overexpressing transgenic mice (Tie1-tTA tetO-Ang2 [where tetO indicates polymeric tetracycline operator], hereafter referred to as Tie1-ANG2 mice) $(38,39)$ at age 8 to 9 weeks, but was weak or absent in normal regions of the vessels (Figure 1C). The amounts of ANG2 and vascu- lar remodeling in Tie1-ANG2 mice were strongly correlated (Figure 1G). Tie1-ANG2 mice off doxycycline from birth had no apparent vascular abnormalities at 3 weeks of age, but at age 25 weeks and older had widespread vascular enlargement accompanied by strong ANG2 immunoreactivity (Supplemental Figure 3, A-C). ANG2 staining was similarly strong at sites of angiogenesis in the trachea of mice conditionally overexpressing VEGF-A (CC1O-rtTA tetO-Vegfa mice [where rtTA indicates reverse tetracycline transactivator], referred to here as CC10VEGF-A mice). In these mice, VEGF-A expression, induced by doxycycline for 7 days (3, 40), promoted the growth of vascular protrusions, but was weak or absent in normal regions of the vasculature (Figure 1D). In all of these models, ANG2 expression was greater at sites of vascular remodeling than in normal blood vessels.

Time course of ANG2 increase in regions of vascular remodeling. To examine the change in ANG2 protein at sites of vascular remodeling over the course of infection, we used $\mathrm{C} 3 \mathrm{H}$ mice because of their greater susceptibility to $M$. pulmonis infection and earlier and more pronounced vascular changes than C57BL/6 mice $(41,44)$. ANG2 increased at sites of vascular remodeling over the course of infection. Strong patches of ANG2 staining were found in focally enlarged vessels at 3 days of infection, but not in neighboring nonremodeled vessels (Figure 2A). ANG2 staining was more intense and uniform in capillaries after 5 to 7 days of infection (Figure 2A). Venules, located between cartilage rings, were enlarged and had uniformly strong ANG2 staining at 3 days (Figure 2, B and C), while corresponding changes in capillaries, located over cartilage rings, were not evident until day 5 (Figure 2, A and C). $\mathrm{C} 3 \mathrm{H}$ mice had significantly greater ANG2 staining and vascular remodeling than $\mathrm{C} 57 \mathrm{BL} / 6$ mice at 7 days of infection (Supplemental Figure 4, A-F).

ANG2 was concentrated in the cytoplasm of endothelial cells at 5 days and longer after infection (Figure 2A). To determine the intracellular distribution of ANG2, we compared ANG2 to P-se- 
A

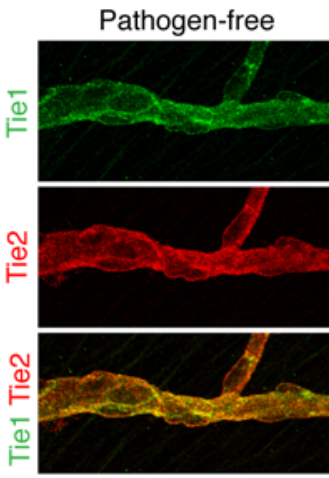

C

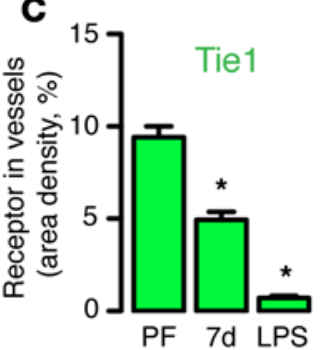

$\mathbf{F}$
M. pulmonis 7d

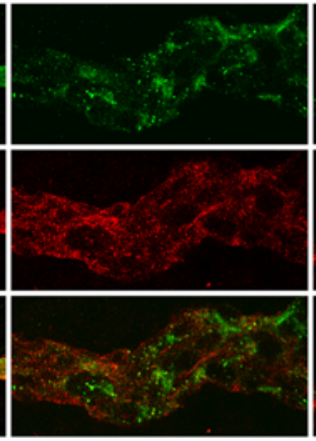

D

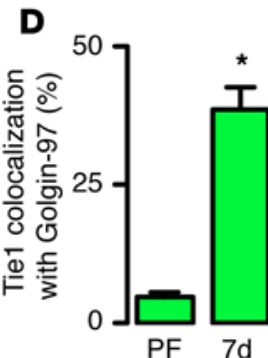

LPS 16h

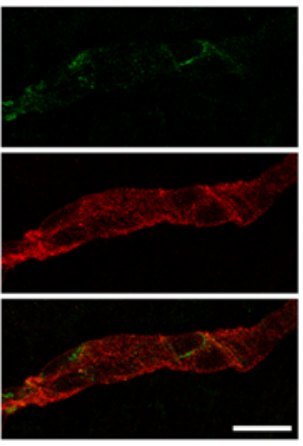

B

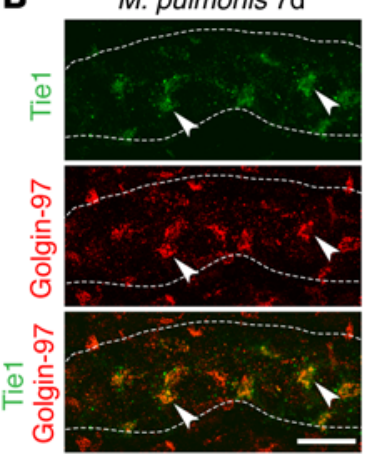

E

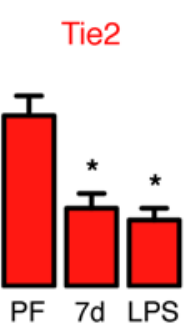

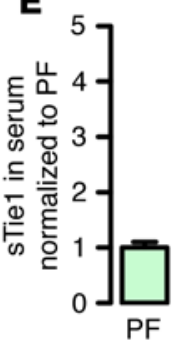

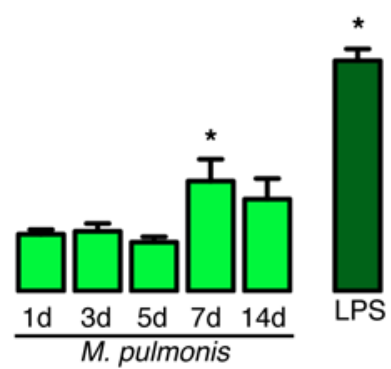

TNF-a 6 h
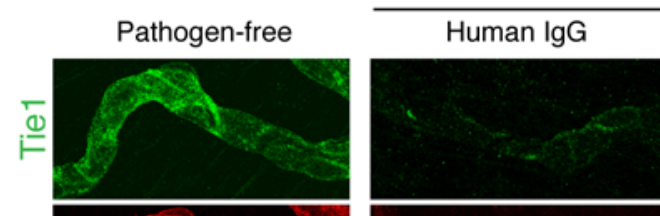

REGN910

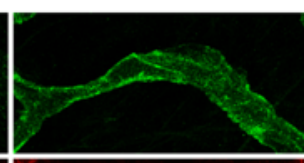

BowANG1
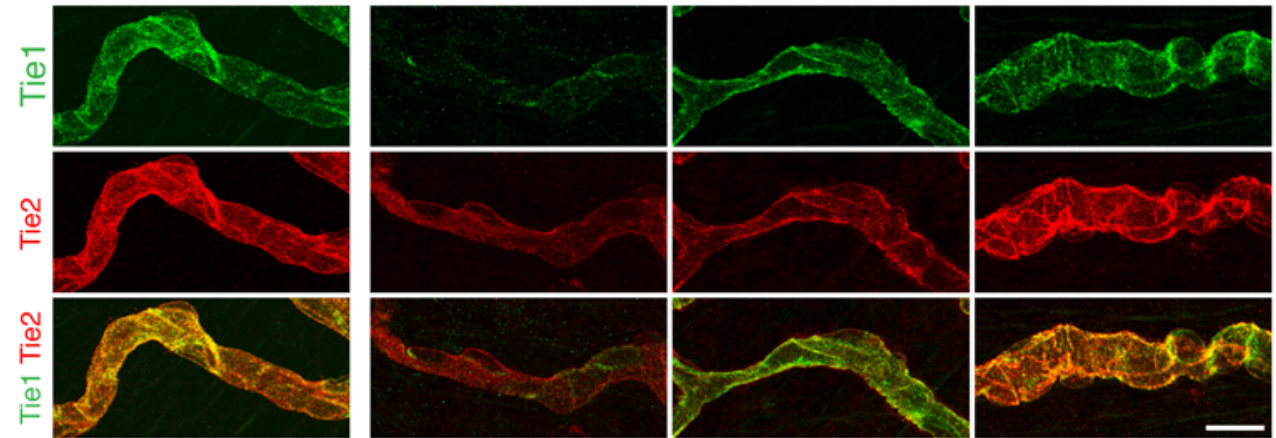

G
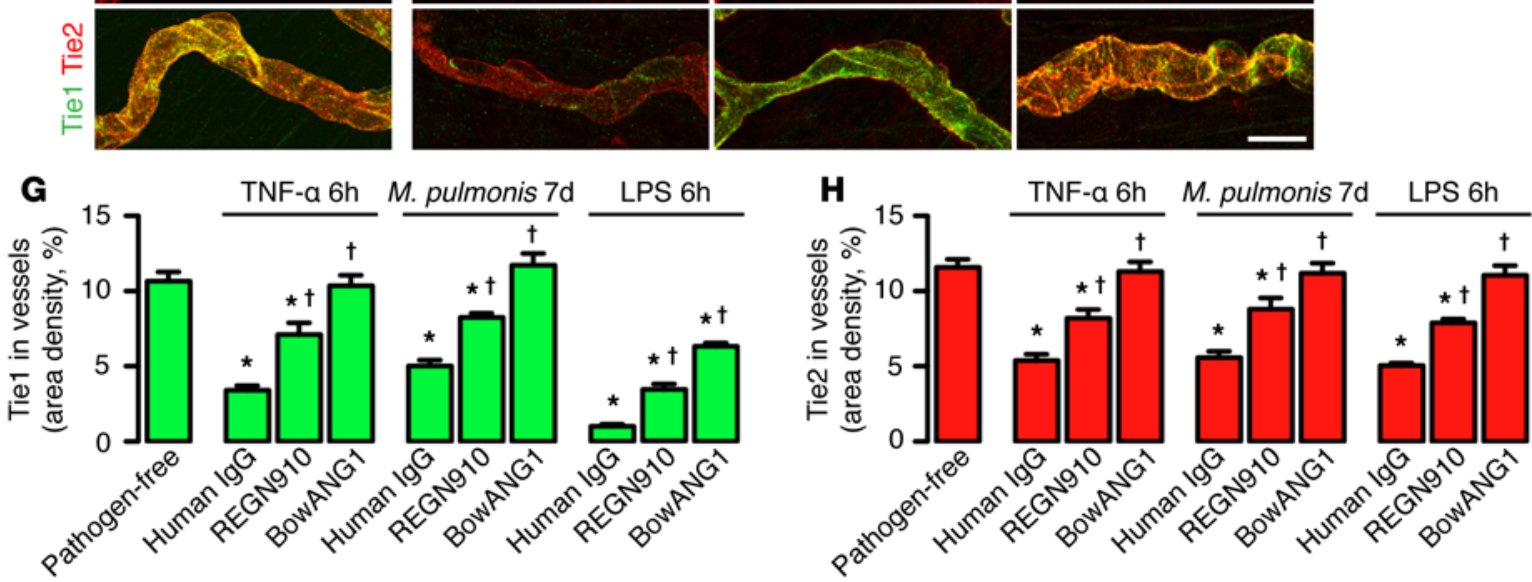

Figure 6. Reduction in endothelial Tie1 and Tie2 after M. pulmonis infection or TNF- $\alpha$. (A) Strong Tie1 and Tie2 staining of tracheal blood vessels under pathogen-free conditions compared with weaker staining after $M$. pulmonis infection for 7 days. LPS for 16 hours (15 mg/kg i.p.) included as a positive control in A, C, and E (38). (B) Strong colocalization of Tie1 with golgin-97 in enlarged vessels after infection for 7 days. (C) Reduction in Tie1 and Tie2 after infection or LPS ( $n=8$ per group). ${ }^{*} P<0.05$ vs. PF controls, 1-way ANOVA. (D) Greater colocalization of Tie1 and golgin- 97 after infection than in pathogen-free controls, assessed by Imaris software ( $n=4$ per group). ${ }^{*} P<0.05$ vs. pathogen-free controls, Student's $t$ test. (E) Comparison of soluble Tie1 in serum at $1,3,5,7$, and 14 days after infection or 16 hours after LPS (15 mg/kg i.p.) normalized to pathogen-free control $\left(n=5-10\right.$ per group). ${ }^{*} P<0.05$ vs. pathogen-free controls, 1-way ANOVA. (F) Strong Tie1 and Tie2 in controls compared with weak staining after TNF- $\alpha$ for 6 hours (0.24 mg/kg i.v.) preceded by control human IgG. Less reduction in Tie1 and Tie2 when TNF- $\alpha$ was preceded by anti-ANG2 antibody REGN910 and no reduction when TNF- $\alpha$ was preceded by Tie2-agonist BowANG1. Scale bars: $20 \mu \mathrm{m}$. (G and $\mathbf{H})$ Tie1 (G) and Tie2 (H) in vessels of groups shown in F, and after infection for 7 days or LPS for 6 hours ( $n=8$ per group). ${ }^{*} P<0.05$ vs. PF controls; ${ }^{\dagger} P<0.05$ vs. corresponding IgG controls, 1 -way ANOVA. 
A

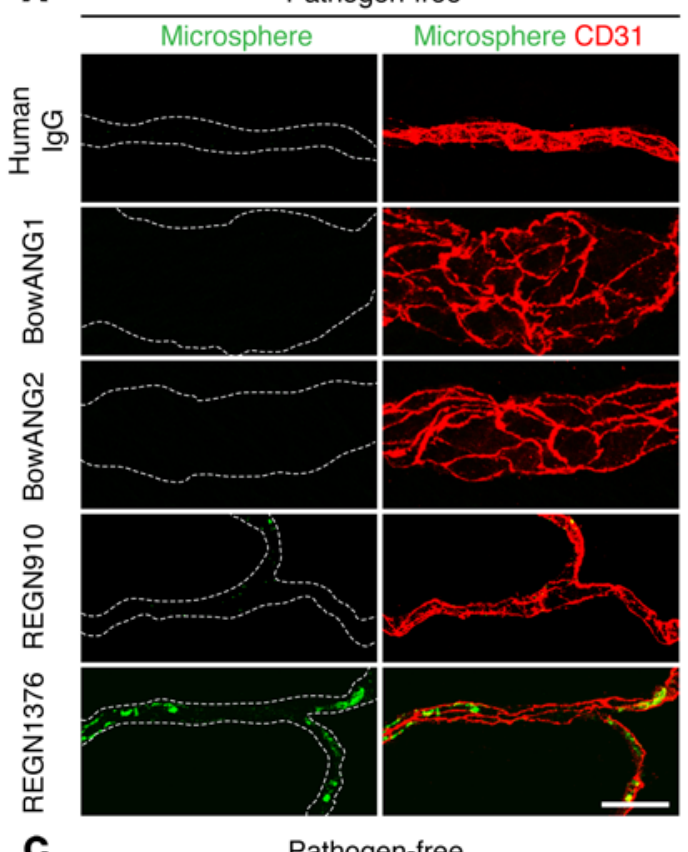

C

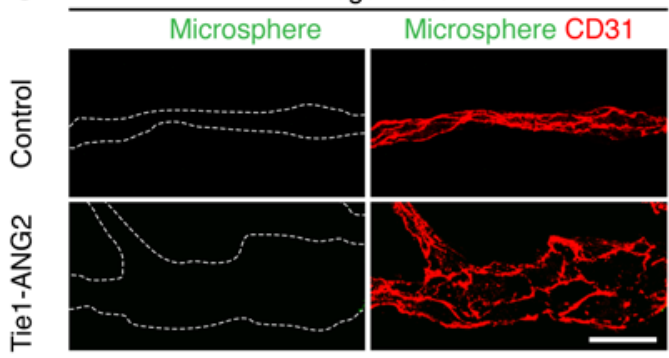

B

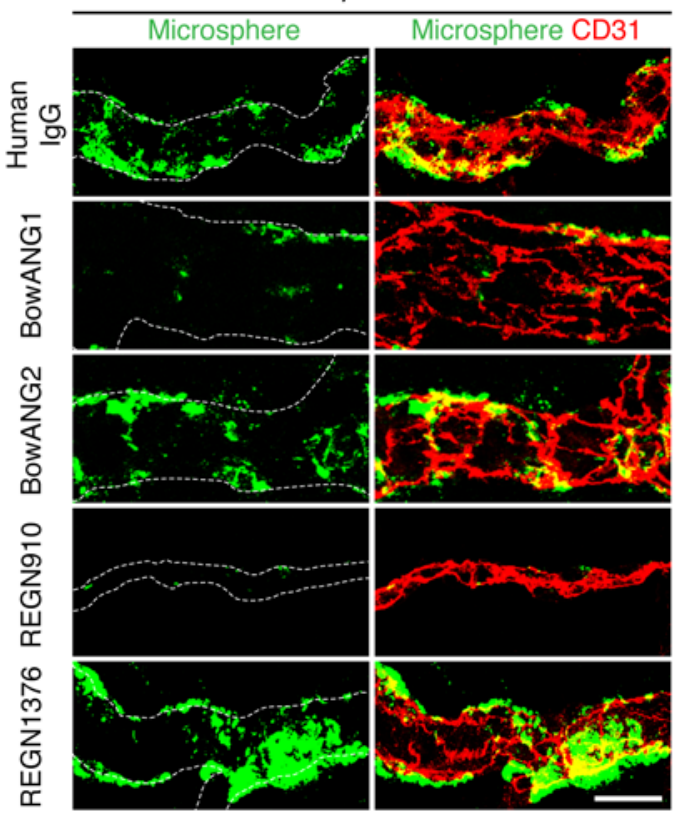

D

M. pulmonis 7d

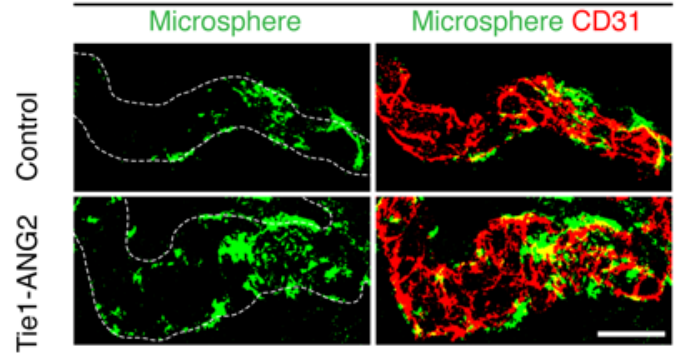

F Pathogen-free M.

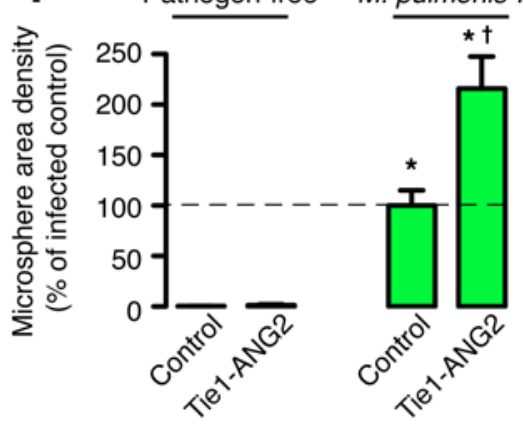

Figure 7. Differences in endothelial barrier function after Tie2 inactivation or overactivation. (A) Comparison of blood vessel leakiness and size in trachea of pathogen-free mice given control antibody (human IgG), BowANG1, BowANG2, anti-ANG2 (REGN910), or anti-Tie2 (REGN1376) for 7 days. BowANG1 and BowANG2 increased vessel size, but only Tie2 blockade resulted in leakage in pathogen-free mice. (B) Similar comparison in mice given the same treatments as in $\mathbf{A}$ but during M. pulmonis infection for 7 days. Vessels were leaky and enlarged in all groups except when ANG2 was blocked, where little leakage or enlargement was found. BowANG1 reduced leakage and exaggerated enlargement, but BowANG2 had comparable leakage with infected controls. (C and D) Comparison of vessel leakiness and size in pathogen-free (C) and infected (D) controls and Tie1-ANG2 mice aged 8 weeks, showing that ANG2 overexpression increased vessel size, but not leakage, under pathogen-free conditions and exaggerated both enlargement and leakage after infection. Dashed white lines delineate vessel borders marked by CD31. Scale bars: $25 \mu \mathrm{m}$. (E) Measurement of leakage in the same groups as shown in $\mathbf{A}$ and $\mathbf{B}(n=4-10$ per group). ${ }^{*} P<0.05$ vs. pathogen-free IgG control; ${ }^{\dagger} P<0.05$ infected with treatment vs. infected IgG control, 1 -way ANOVA. (F) Measurement of leakage in the same groups as shown in $\mathbf{C}$ and $\mathbf{D}\left(n=4-8\right.$ per group). ${ }^{*} P<0.05$ vs. pathogen-free control; ${ }^{\dagger} P<0.05$ infected Tie1-ANG2 vs. infected control, 1 -way ANOVA.

lectin and VWF in Weibel-Palade bodies and to golgin-97 (Golgal gene) in the Golgi complex. ANG2 is known to associate with vWF, but not with P-selectin, in cultured endothelial cells (28). Indeed, we found little colocalization of ANG2 with P-selectin under either pathogen-free or infected conditions (Supplemen- tal Figure 5A and Figure 2, D and E). The small amount of ANG2 present under pathogen-free conditions colocalized with vWF, indicative of ANG2 storage in Weibel-Palade bodies, but not with golgin-97 (Supplemental Figure 5A), indicative of greater storage than production. After infection, ANG2 colocalized strongly with 
A
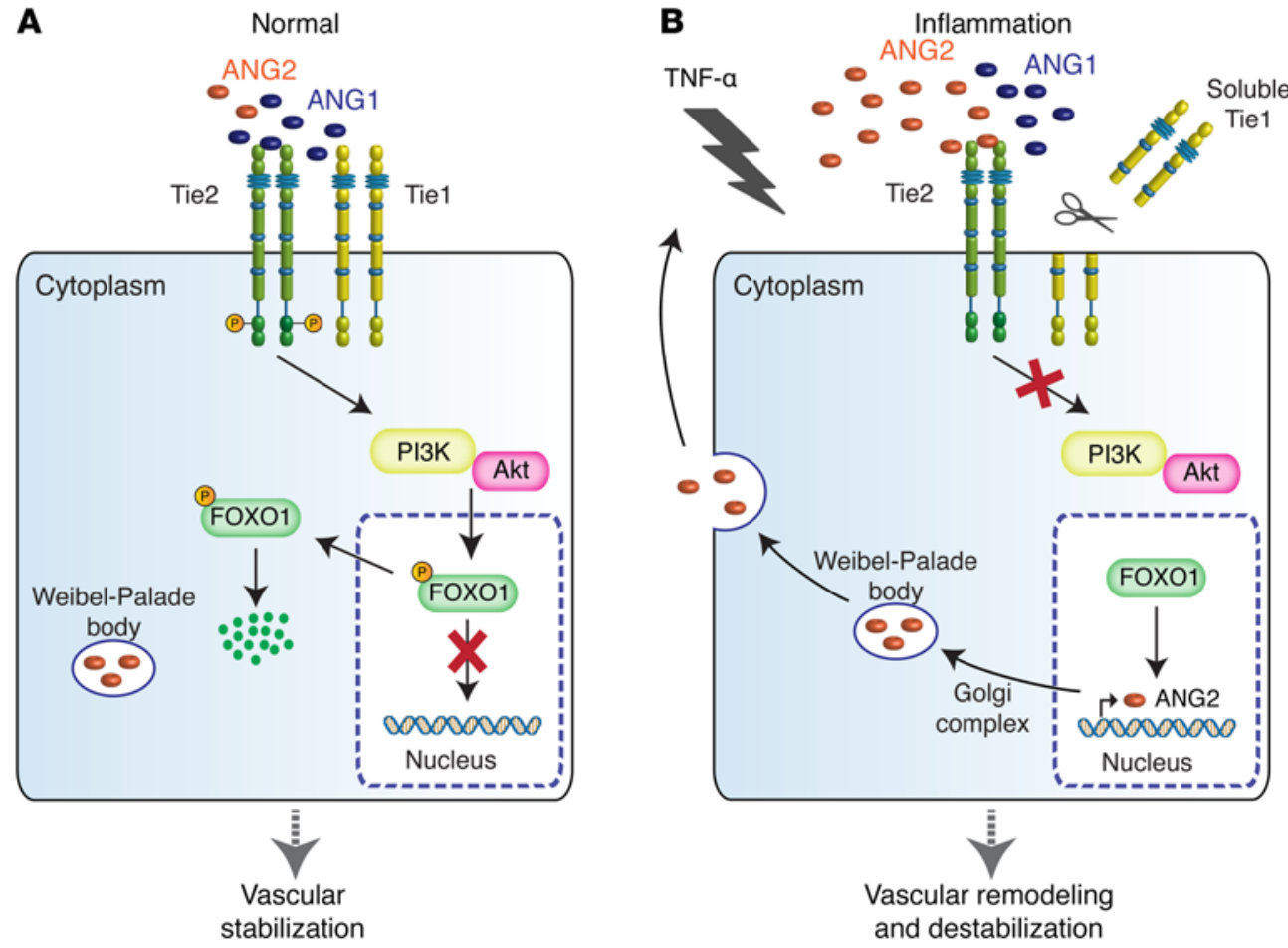

Figure 8. Diagram of regulatory loop linking ANG1, ANG2, Tie1, and Tie2 in endothelial cells. (A) Under normal conditions, the agonist action of ANG1 dominates ANG2 in activating Tie2 signaling in a Tie1-dependent manner (38), leading to PI3K/Akt pathway activation. Akt-dependent phosphorylation of FOXO1 suppresses transcriptional function by promoting nuclear exclusion, ubiquitination, and degradation, which increases expression of genes that promote vascular stabilization. (B) After M. pulmonis infection, TNF- $\alpha$ is released, Tie1 is reduced by ectodomain shedding, and Tie2 phosphorylation is suppressed by ANG2 secreted from Weibel-Palade bodies. $\mathrm{PI3K} /$ Akt pathway inactivation promotes FOXO1 transcriptional activity and increases expression of ANC2 and other vascular destabilizing genes. The antagonistic action of greater ANG2 dominates the agonist action of ANG1, maintains suppression of Tie2 signaling, and establishes a positive feedback loop, with sustained Akt inactivation, FOX01 transcriptional activation, and ANG2 production. Vascular destabilization, remodeling, and leakiness are downstream consequences of this ANG2-Tie2 regulatory loop after infection.

golgin-97, but weakly with vWF (Figure 2, D and E), consistent with greater ANG2 production and release with limited storage in Weibel-Palade bodies.

Link between ANG2 antagonism of Tie2 and vascular remodeling. The mechanistic involvement of ANG2 in the regulation of Tie2 signaling during vascular remodeling was assessed by examining changes in Tie2 phosphorylation, with or without pharmacologic inhibition of ANG2 or Tie2. Endothelial p-Tie2 was strong in tracheal blood vessels under pathogen-free conditions (Figure 3A), as in normal blood vessels of other organs (Supplemental Figure 5B) (45). No change in p-Tie 2 was detected after inhibition of ANG2 by REGN910 (24) under pathogen-free conditions, but inhibition of Tie2 signaling by the function-blocking antibody REGN1376 reduced p-Tie2 (Figure $3 \mathrm{~A}$ and Supplemental Figure 6, A-C). Neither inhibition of ANG2 nor inhibition of Tie2 resulted in a change in vessel size under these baseline conditions (Figure 3A and Supplemental Figure 6, A-C).

In contrast, $\mathrm{p}$-Tie 2 was conspicuously reduced and vessels were enlarged at 7 days after infection (Figure 3B). Staining for $\mathrm{p}$-Tie2 at tyrosine 1100 (Y1100), a site required for activation of PI3K/Akt signaling through association with the PI3K p85 regulatory subunit $(46,47)$, was similarly suppressed in remodeled vessels after infection (Supplemental Figure 7A). ANG2 blockade by REGN910 prevented the decrease in $\mathrm{p}$-Tie 2 and vascular enlarge- ment (Figure 3, B-D). Furthermore, inhibition of Tie2 during infection was accompanied by even weaker $\mathrm{p}$-Tie2 and more vascular enlargement (Figure 3, $\mathrm{B}-\mathrm{D})$. These changes were still present when ANG2 was inhibited together with Tie2 during infection (Figure 3, B-D). These findings demonstrate that inhibition of ANG2 prevented the suppression of $\mathrm{p}$-Tie 2 and vascular remodeling during infection and that inhibition of Tie2 had the opposite effect.

We next asked whether suppression of p-Tie2 during infection could be overcome by administration of the ANG1 mimic BowANG1. This experiment revealed that $\mathrm{p}$-Tie2 remained strong in the presence of BowANG1 during infection, but vascular enlargement was exaggerated (Figure 3, B-D). BowANG1 had similar effects on $\mathrm{p}$-Tie2 and vessel size in pathogen-free mice (Supplemental Figure $6, \mathrm{~A}^{-} \mathrm{C}$ ), where the enlargement was similar to that in reports of ANG1 overexpression $(35,48-50)$. ANG2 blockade by REGN910 did not change the effects of BowANG1 on vascular enlargement under pathogen-free conditions or after infection (Figure 3, B-D, and Supplemental Figure 6, A-C).

The temporal sequence of Tie2 suppression and increase in ANG2 after infection was determined by examining earlier time points. No change was found in p-Tie2 or ANG2 staining at 1 day or 2 days after infection, regardless of whether ANG2 was inhibited by REGN910 (Supplemental Figure 7, B and C). However, at 3 days, when ANG2 was still low, p-Tie2 was weaker than normal and had a uniformly punctate appearance, unlike the strong junctional staining for $\mathrm{p}$-Tie2 in pathogen-free controls (Figure 3, E and $\mathrm{F})$. Here, ANG2 inhibition prevented the decrease in $\mathrm{p}$-Tie2 (Figure 3, E and F). These findings indicate that the reduction in $\mathrm{p}$-Tie2 preceded the increase in ANG2 after infection.

FOXO1 regulation of ANG2 expression after infection. We next sought to identify the link between Tie2 activity and ANG2 expression in endothelial cells. We explored evidence that Tie2 activation promotes PI3K/Akt signaling and consequent FOXO1 transcriptional inactivation and that FOXO1 activation promotes ANG2 expression in endothelial cells $(10,11,13)$. Under pathogen-free conditions, little or no nuclear FOXO1 or ANG2 staining was evident in endothelial cells of tracheal blood vessels, indicating low FOXO1 transcriptional activity and low ANG2 expression under normal conditions (Figure 4A). However, after infection, nuclear 
FOXO1 and cytoplasmic ANG2 were strong in the remodeled vessels (Figure 4B), which had low p-Tie2 (Figure 3B). Consistent with suppression of Tie2/PI3K/Akt signaling, FOXO1 and ANG2 were even stronger when PI3K was further inhibited by treatment with BKM120 during the infection (Supplemental Figure 8, A-D). These findings align with evidence that nuclear FOXO1 and ANG2 are linked and with the concept that the increase in ANG2 after infection is a consequence of FOXO1 transcriptional activation resulting from suppression of Tie2/PI3K/Akt signaling in endothelial cells.

We then determined whether ANG2 antagonism of Tie2 was required for the increase in nuclear FOXO1 and ANG2 in endothelial cells after infection. We found that administration of REGN910 to block ANG2 during infection inhibited nuclear accumulation of FOXO1, expression of ANG2, and vascular enlargement (Figure 4, B and C), consistent with maintenance of $\mathrm{p}$-Tie2 in the presence of REGN910 (Figure 3, B and C).

Consistent with these findings, blood vessels of infected mice that received BowANG1 had little or no nuclear FOXO1 or ANG2, but were enlarged even more than the untreated controls (Figure 4, B and C). When BowANG1 was combined with ANG2 inhibition by REGN910 during infection, blood vessels had little nuclear FOXO1 or ANG2 (Figure 4C); this resembled the low levels found in pathogen-free mice (Supplemental Figure 6, D-F). These findings indicate that the increase in nuclear FOXO1 and ANG2 expression in vascular remodeling during infection can be prevented by blocking ANG2 or by giving exogenous ANG1. The results implicate an essential role of ANG2 antagonism of Tie2 in FOXO1 activation and increased ANG2 expression after infection.

We next compared the effect of ANG2 overexpression on FOXO1 in vessels of pathogen-free and infected mice. After BowANG2, nuclear FOXO1 was weak or absent in enlarged vessels in pathogen-free mice (Supplemental Figure 9, A and C). Similarly, ANG2 overexpression and vascular enlargement in pathogen-free Tie1-ANG2 mice were accompanied by weak or absent nuclear FOXO1 (Supplemental Figure 9, B and C). These findings are consistent with previous evidence that ANG2 overexpression can induce vascular enlargement in otherwise normal mice by activating Tie2 $(30,31)$. After infection, nuclear FOXO1 was greater and ANG2 staining was stronger in remodeled vessels of Tie1-ANG2 mice than in control mice (Figure 4, D and E). Infection of Tie1-ANG2 mice was accompanied by significantly higher mortality (Figure 4F). Importantly, the exaggerated vascular changes in infected Tie1-ANG2 mice were prevented by REGN910 blockade of ANG2 (Figure 4, G and H), indicating that Ang2 overexpression amplified the response to infection. Overall, these findings document the essential role of ANG2 antagonism of Tie2 in FOXO1-driven ANG2 expression and vascular remodeling during infection.

To assess the specificity of nuclear FOXO1 as a readout for induction of ANG2 expression in endothelial cells under the conditions tested, we also examined FOXO3A, a related transcription factor in endothelial cells involved in angiogenesis (13). Although the amount of nuclear FOXO3A was much greater after infection, most was in cells other than endothelial cells (Supplemental Figure 9D).

Mechanism of context-dependent action of ANG2 on Tie2 signaling. With the goal of reconciling the contextual agonist/antagonist action of ANG2 on Tie2 $(15,51,52)$, we asked whether ANG2 acts as a Tie2 agonist under pathogen-free conditions but as an antagonist after M. pulmonis infection. We learned that the ANG2 mimic BowANG2 (21) promoted Tie2 phosphorylation and vascular enlargement in pathogen-free mice (Figure 5, A and C); this was similar to - but weaker than - the results with the Tie2 agonist BowANG1. To determine whether the amount of ANG2 influenced its action on Tie2, we tested a range of doses of BowANG2 on p-Tie2 in pathogen-free mice. BowANG2 acted as a Tie2 agonist over the entire dosage range and did not suppress $\mathrm{p}$-Tie2 at any dose tested in pathogen-free mice (Supplemental Figure 10, A-C). By comparison, BowANG2 given during infection reduced p-Tie2, as was found in infected mice without BowANG2, consistent with ANG2 antagonism of Tie2 (Figure 5, B and C). As further support, this context-dependent effect of BowANG2 was mimicked by genetic overexpression of ANG2 in Tie1-ANG2 mice (Figure 5, A-D). Unlike BowANG2, BowANG1 promoted Tie2 activation, regardless of whether the mice were pathogen-free or infected (Figure 5, A-D).

To examine the mechanism by which infection promoted ANG2 antagonism of Tie2, we built on evidence that Tie1 is essential for agonistic action of ANG2 on Tie2 (38). The finding that Tie 1 in endothelial cells rapidly decreases after LPS exposure (38) led us to ask whether Tie1 also decreases after M. pulmonis infection. Indeed, Tie1 immunofluorescence was strong in the plasma membrane under pathogen-free conditions, but was weak at 3 days and nearly absent at 7 days after infection (Figure 6, A and C, and Supplemental Figure 11, A and B). Despite the absence of staining in the plasma membrane at 7 days, Tie1 had a patchy distribution in the cytoplasm, which colocalized with the Golgi apparatus marker golgin-97 (Figure 6, B and D). At 14 days after infection, Tie1 was stronger than at 7 days and close to basal levels (Supplemental Figure 11, A and B), indicative of receptor recovery after the initial reduction.

The ectodomain of Tie1 undergoes proteolytic cleavage in response to TNF- $\alpha$ and other inflammatory cytokines in vitro (53). Similarly, LPS exposure in vivo leads to sloughing of the Tie1 ectodomain, which can be measured as soluble Tie1 in blood (38). We found that soluble Tie1 in blood increased to twice the baseline at 7 days of M. pulmonis infection and was still elevated but less so at 14 days (Figure 6E).

Tie2 in the tracheal vasculature decreased along with Tie1 during the first week after infection (Figure 6, A and C, and Supplemental Figure 11, A and C). The reduction in Tie2, however, occurred more gradually than the decrease in Tie1 (Supplemental Figure 11). The initial reduction of Tie 2 after infection resembled the change after LPS (38), as confirmed here (Figure 6, A and C). Like Tie1, Tie2 recovered as the infection evolved and was greater at 14 days than at 7 days (Supplemental Figure 11, A and C).

Because TNF- $\alpha$ increases many-fold and promotes vascular remodeling during $M$. pulmonis infection in mice $(2,17)$ and can also induce Tie1 ectodomain shedding in endothelial cells (53), we asked whether TNF- $\alpha$ could mimic the reduction in Tie1 and Tie2 found after infection. Examination of a range of doses revealed that TNF- $\alpha$ at a dose of $0.24 \mathrm{mg} / \mathrm{kg}$ i.v. reduced Tie1 and Tie2 (Supplemental Figure 12, A and B), which fit with increased soluble Tie1 found in the blood after TNF- $\alpha$ was administered (38). The reduction after TNF- $\alpha$ administration resembled but was less than the reduction after the high dose of LPS ( $15 \mathrm{mg} / \mathrm{kg}$ i.p.). 
Evidence of synergistic effects of inhibiting ANG2 and TNF- $\alpha$ together in suppressing vascular remodeling after M. pulmonis infection (17) led us to ask whether ANG2 sensitizes endothelial cells to the actions of TNF- $\alpha$. Using Tie1 and Tie 2 as readouts in vivo, we found that TNF- $\alpha$-induced reduction of both receptors was less when ANG2 was blocked by REGN910 (Figure 6, F-H). To test whether ANG2 blockade unmasked agonistic effects of ANG1, we asked whether sustained activation of Tie2 by exogenous ANG1 could protect against TNF- $\alpha$-mediated reduction of Tie1 and Tie2. Indeed, BowANG1 fully prevented the reduction of both receptors (Figure 6, F-H). Similarly, ANG2 inhibition by REGN910 or Tie2 activation by BowANG1 reduced the suppression of Tie1 and Tie2 after M.pulmonis infection or LPS (Figure 6, G and H, and Supplemental Figure 12, C and D). Together, the findings implicate TNF- $\alpha-$ driven reduction of Tie 1 by ectodomain shedding as a promoter of ANG2 antagonism of Tie2 in these models of inflammation.

Differences in endothelial barrier function after Tie2 inactivation or overactivation. When vascular enlargement is driven by Tie2 overactivation, vessels are resistant to leakage, but when it is driven by Tie 2 inhibition, they are typically unstable and leaky $(16,18$, $19,32,37,54)$. We exploited this background to distinguish vascular remodeling under opposite states of Tie 2 activity in our model, using leakage of 50-nm fluorescent microspheres as a readout (55).

We first asked whether barrier function changed after administration of BowANG1 or BowANG2 under baseline conditions. No evidence of leakage was found, as in the controls, but vascular enlargement was conspicuous (Figure 7, A and E), as reported previously $(32,37,50)$. ANG2 blockade by REGN910 had no effect on leakage under baseline conditions. However, inhibition of Tie2 by REGN1376 was accompanied by a modest but significant increase in microsphere extravasation without vascular enlargement (Figure 7, A and E). The absence of effect of ANG2 blockade fits with no discernible role of ANG2 under normal conditions, but the presence of leakage after Tie2 blockade is consistent with the essential involvement of constitutive ANG1 activation of Tie2 in the maintenance of endothelial barrier function.

Effects of these manipulations of Tie 2 signaling changed after infection, where remodeled vessels were more leaky (Figure 7B). BowANG1 reduced the leakage and exaggerated the vascular enlargement (Figure 7, B and E), consistent with earlier findings (37). Leakage after BowANG2 was comparable to that in infected controls (Figure 7, B and E), where ANG2 expression was already high. ANG2 blockade by REGN910 reduced leakage (Figure 7, B and E), indicating that the antileak effect of endogenous ANG1 was unmasked by inhibiting ANG2. By comparison, leakage after infection was exaggerated when Tie2 was blocked by REGN1376 (Figure 7, B and E). Leakage after infection reflected a shift toward ANG2 dominance in the balance between ANG1 agonism and ANG2 antagonism of Tie2 signaling.

To gain further insight into the action of ANG2 in vascular destabilization and leakage, we compared the effect of ANG2 overexpression at baseline and after infection. ANG2 overexpression in pathogen-free Tie1-ANG2 mice was accompanied by vascular enlargement without leakage (Figure 7, C and F), which is similar to the Tie2 agonist effects of BowANG2 (Figure 7A). However, leakage in Tie1-ANG2 mice was exaggerated after infection (Figure 7, D and F).
These findings support evidence that ANG2 acts as a Tie2 agonist under baseline conditions, but acts as a Tie2 antagonist after infection. Also, the findings demonstrate that both ANG1 and ANG2 act as Tie2 agonists and promote vascular enlargement and stability under otherwise normal conditions, consistent with previous reports $(16,20,32,37)$, but also that, in inflammation, ANG2 acts as a Tie2 antagonist and promotes vascular destabilization and leakage.

\section{Discussion}

The goal of this study was to reconcile 2 paradoxes that have long been recognized about the action of ANG2 on blood vessels: (a) ANG2 can inhibit or activate Tie2, depending on the context, and (b) vascular remodeling can result from either inactivation or overactivation of Tie2. We explored the actions of ANG2 as a Tie2 agonist or antagonist by manipulating the actions of ANG2 on Tie2 signaling and the links among Tie1, Tie2, FOXO1, ANG2 expression, and vascular remodeling. We found that under baseline conditions, ANG2 overexpression promoted vascular remodeling by acting as a Tie2 agonist: vessels had high p-Tie2, low nuclear FOXO1, and no leakage. In contrast, after M. pulmonis infection, ANG2 antagonism of Tie 2 promoted vascular remodeling: vessels had low p-Tie2, high nuclear FOXO1, high ANG2 protein, and leakage. Nuclear FOXO1 and cytoplasmic ANG2 were further increased by inhibiting PI3K/Akt signaling during infection. Mechanistic studies revealed that the infection or TNF- $\alpha$ triggered reduction in Tie1 by ectodomain shedding and promoted ANG2 antagonism of Tie2. Together, the results indicate that under normal conditions, ANG2 agonism of Tie 2 promotes enlarged stable vessels, in a manner similar to but weaker than the effects of ANG1. However, when Tie1 is reduced in inflammation, ANG2 antagonism of Tie2 initiates a positive feedback loop through FOXO1-driven ANG2 expression that leads to vascular remodeling.

Opposing actions of ANG2 on Tie2 are governed by the presence or absence of inflammation. ANG2 is well documented as an antagonist of Tie2 signaling in endothelial cells (15, 51, 52, 56). However, ANG2 can also function as a weak agonist of Tie2 signaling under conditions including absence of ANG1 $(23,57)$, high ANG2 concentration, and prolonged ANG2 exposure (20, 58-60). Vascular enlargement after ANG2 overexpression has previously been reported in ANG2 gain-of-function studies of otherwise healthy mice (31). Our study revealed that ANG2 overexpression in Tie1-ANG2 mice led to strong p-Tie2 at sites of vascular enlargement under normal conditions, which fits with ANG2 acting as a Tie2 agonist $(20,58)$.

Unlike its agonistic action on normal blood vessels, ANG2 antagonized Tie2 in the inflamed vasculature. Inhibition of ANG2 by REGN910 prevented the antagonism, and thereby Tie 2 activation was maintained despite the presence of infection, presumably by endogenous ANG1. Reductions in Tie1 and Tie 2 after infection or TNF- $\alpha$ challenge were similarly less after REGN910. Further, administration of BowANG2 or genetic overexpression of ANG2 in Tie1-ANG2 mice suppressed p-Tie2 after infection, but had the opposite effect under normal conditions. These findings, together with evidence of rapid release of ANG2 from tracheal blood vessels after inflammatory stimuli (38), support a mechanism whereby the initial reduction in $\mathrm{p}$-Tie2 triggers a positive feedback loop that increases ANG2 expression and maintains p-Tie2 suppression. 
The use of pharmacological inhibition of ANG2 by REGN910 circumvented the confounding effects of impaired immune responses, lymphatic defects, and other abnormalities in ANG2 null mice (61-64). The anti-ANG2 antibody also permitted control over onset, duration, and magnitude of antagonism. Effects of ANG2 blockade by REGN910 were greater than found previously in the M. pulmonis model after anti-ANG2 antibody AZD5180 (17), probably due to the lower $K_{D}$ for mouse ANG2 of REGN910 $(0.09 \mathrm{nM})$ than AZD5180 (3 nM) despite their similar affinities for human ANG2 $(24,65)$.

Multiple lines of evidence indicate that the actions of angiopoietins on Tie 2 signaling are strongly influenced by the interaction of Tie1 with Tie $2(9,38)$. Deletion of endothelial Tie1 prevents ANG1-induced Tie2 activation and resultant vascular enlargement in otherwise normal mice (38). Our studies revealed that the decrease in Tie1 in blood vessels after M. pulmonis infection was accompanied by increased levels of soluble Tie1 in blood as a reflection of ectodomain shedding. The reduction in Tie1 after infection was also accompanied by suppression of $\mathrm{p}$-Tie2, consistent with Tie1 as a positive regulator for Tie 2 activation. Although Tie1 protein in endothelial cells decreased after infection, the Tie1 promoter continued to drive ANG2 expression in Tie1-ANG2 mice, which had even stronger ANG2 staining after infection. Further studies are needed to understand the links between the phosphorylation states of Tie1 and Tie 2 and the relationship between Tie1 ectodomain shedding and Tie1 expression.

TNF- $\alpha$, which increases 6 -fold or more after infection $(2,17)$ and is implicated in the reduction of endothelial cell Tie1 by ectodomain shedding, promoted ANG2 antagonism of Tie2. TNF- $\alpha$ injected i.v. was followed by reductions of both Tie1 and Tie2 in endothelial cells, which resembled the changes after M. pulmonis infection or LPS. Importantly, both effects were reduced by ANG2 blockade with REGN910 and prevented by sustained Tie2 activation with BowANG1. Evidence that ANG2 and TNF- $\alpha$ act synergistically as drivers of vascular remodeling after $M$. pulmonis infection (17) is indicative of ANG2 sensitization of endothelial cells to the effects of TNF- $\alpha$. The findings also indicate that sustained Tie2 activation by ANG1 is sufficient to protect against TNF- $\alpha$ suppression of Tie 1 and Tie 2 in endothelial cells. These features fit with the known balance of Tie 2 agonists and antagonists as opposing regulators of vascular stability and remodeling $(7,37)$.

ANG2 binding to integrins has been reported to contribute to effects of ANG2 independent of Tie2, where Tie2 is downregulated in endothelial cells $(66,67)$. Our evidence that the effects of blocking ANG2 during infection were completely abolished by inhibiting Tie2 does not exclude a contribution of integrins, but does emphasize the importance of ANG2/Tie2 interactions in the vascular changes.

Positive feedback loop between ANG2 antagonism of Tie2 and ANG2 production. The importance of Tie2 signaling in vascular remodeling has long been recognized, and mechanistic links between Tie2 activity and ANG2 expression in endothelial cells are beginning to be elucidated. As a downstream target of Tie2 signaling, FOXO1 regulates ANG2 expression and is essential for embryonic development $(11,13,68)$. Activation of Tie2/Akt signaling reduces FOXO1 transcriptional activity by phosphorylation and nuclear exclusion, which limits ANG2 production through negative feedback (20).
Our finding of strong p-Tie2 and low ANG2 expression after ANG2 inhibition in infected mice led us to test the hypothesis that increased expression of ANG2 in inflammation is a consequence of ANG2 antagonism of Tie2 signaling. In support of this hypothesis, ANG2 increased preferentially in endothelial cells that had low p-Tie2 and high nuclear (activated) FOXO1 in enlarged vessels. The changes in FOXO1 and ANG2 during infection were exaggerated by inhibition of PI3K/Akt signaling. Further, ANG2 blockade prevented the increase in nuclear FOXO1 and ANG2, which could be explained by unmasking ANG1 activation of Tie2 and consequent suppression of FOXO1 transcriptional activity. The results fit with an initial release of stored ANG2 soon after infection, triggering a positive feedback loop, where p-Tie 2 suppression is followed by FOXO1 activation and upregulation of ANG2 in endothelial cells. Reduced colocalization of ANG2 with vWF in Weibel-Palade bodies after infection is consistent with greater release than storage of newly produced ANG2. Together, the findings revealed a previously unknown positive feedback loop, where ANG2 antagonism of Tie2 during infection promoted FOXO1-driven expression of ANG2 and vascular remodeling (Figure 8).

In addition to Tie2, VE-cadherin is known to regulate PI3K/ Akt activity and downstream effects of FOXO1 (69). The transcriptional coactivator Yes-associated protein (YAP), another downstream target of the PI3K/Akt pathway, regulates ANG2 expression in response to VE-cadherin-mediated endothelial cell contacts (70). FOXO1 also regulates a large set of genes involved in angiogenesis and vascular remodeling $(11,13)$. Additional studies are needed to develop a more complete understanding of ANG2 collaboration with other FOXO1 or YAP target genes to regulate vascular changes in inflammation.

Vascular enlargement under opposing states of Tie2 activity: stability versus leakiness. Our findings confirm that vascular remodeling can result from either overactivation or inactivation of Tie2. The surprise was that ANG2 acted as a Tie2 agonist when administered as a recombinant protein (BowANG2) or was genetically overexpressed (Tie1-ANG2 mice) in otherwise normal conditions, but was an antagonist after M.pulmonis infection. It is known that when Tie2 is inactivated after infection, vessels enlarge and become leaky as a consequence of endothelial cell proliferation and loss of junctional stability $(4,42)$. ANG2 and TNF- $\alpha$ contribute to these changes (17, 40). FOXO1 transcriptional activation leads to decreased expression of the tight junction protein claudin -5 and reduced junction stability $(11,69)$. These changes could result in loss of endothelial cell contact inhibition, activation of cell proliferation, and vascular enlargement (71). Mechanistic links between Tie2 inactivation and endothelial cell proliferation deserve further study.

Vascular remodeling can also occur with activating Tie 2 mutations or ANG1 or ANG2 overexpression $(31-34,38)$. Tie2 activation by ANG1 overexpression promotes circumferential vascular enlargement that results from VEGF-independent endothelial cell proliferation without sprouting (49). ANG1 is a weak mitogen compared with VEGF $(47,72)$ but is a potent endothelial cell survival factor. ANG1 activation of Tie 2 can promote endothelial cell mitogenic activity through MAPK and PI3K $(12,73)$ or Erk1/2 signaling (74). Effects of Tie2 activation depend on which tyrosine residues are phosphorylated (47), but the links to blood vessel growth and stability are unclear. 
Blood vessels that underwent remodeling under opposite states of Tie2 phosphorylation could be distinguished by differences in endothelial barrier function. Antileak effects of Tie2 activation and proleak effects of Tie2 suppression fit with many reports of the stabilizing actions of ANG1 and destabilizing actions of $\operatorname{ANG} 2(16,18,19,32,37,54)$.

In conclusion, the present study confirms that ANG2 can promote vascular remodeling by acting as a Tie2 agonist or antagonist, but the opposing actions occur under different circumstances and have different consequences (Figure 8). Under normal conditions, ANG2 - particularly when overexpressed or at high concentration - acts as a Tie 2 agonist that promotes vessel enlargement and stability, but is less potent than ANG1. In contrast, in the presence of inflammation accompanying $M$. pulmonis infection, ANG2 antagonism of Tie2 activates FOXO1 transcriptional activity, increases ANG expression, and initiates a positive feedback loop that leads to enlarged, leaky vessels. Infection or TNF- $\alpha$ triggers reduction in Tie1 by ectodomain shedding and promotes ANG2 antagonism of Tie2. Our study highlights the importance of identification of upstream regulators of the responsiveness of Tie 2 to angiopoietins under diverse conditions. Yet ANG2 and Tie1/Tie2, acting in synergy with TNF- $\alpha$ and other inflammatory cytokines, are clearly important vascular modulators and biomarkers in disease.

\section{Methods}

Mice. Ang2-EGFP transgenic breeder mice (Tg [Ang2-EGFP] DJ90Gsat/Mmcd) were purchased from the Mutant Mouse Regional Resource Centers (MMRRC). Ang2-EGFP mice were created by inserting the EGFP-coding sequences under the Ang2 promoter in an Ang2-containing BAC (RP23-112L4) through homologous recombination by the Gene Expression Nervous System Atlas (GENSAT) BAC transgenic project (43). This BAC contains an approximately 187-kb-long mouse genomic contig, spanning from approximately $60 \mathrm{~kb}$ upstream of the Ang2 start codon to $127 \mathrm{~kb}$ downstream of the start codon.

Tie1-ANG2 (Tie1-tTA tetO-Ang2) mice were generated by breeding Tie1-tTA driver mice $(30,39)$ with tetO-Ang2 responder mice (38). Endothelial-specific overexpression of ANG2 was induced in Tie1ANG2 mice by discontinuation of doxycycline after birth. Doxycycline (Sigma-Aldrich, $2.0 \mathrm{mg} / \mathrm{ml}$ in $5 \%$ sucrose) was given in the drinking water of pregnant mice from the start of mating until birth. Single transgenic mice were used as controls.

CC10-VEGF-A (CC1O-rtTA tetO-Vegfa) mice were generated at Yale University (New Haven, Connecticut, USA), as described (40, 75). Briefly, 2 constructs were used containing the CC10 promoter and rtTA, and tetO with human VEGF. Male transgenic mice were bred with wild-type C57BL/6 female mice. VEGF-A overexpression in club cells and alveolar type II cells of the airways and lung of CC10-VEGF-A mice was induced by administration of doxycycline $(1 \mathrm{mg} / \mathrm{ml}$ in $5 \%$ sucrose) in the drinking water (40). Wild-type mice were used as controls. Wild-type pathogen-free female mice were purchased from Jackson Laboratory (C57BL6/J) or Simonsen Laboratories $(\mathrm{C} 3 \mathrm{H})$.

All mice were housed under barrier conditions and studied at 8 to 10 weeks of age unless otherwise specified. Mice were anesthetized by intramuscular injection of ketamine $(87 \mathrm{mg} / \mathrm{kg})$ and xylazine $(13 \mathrm{mg} /$ $\mathrm{kg}$ ) before experimental procedures.
M. pulmonis infection and other inflammation models. Anesthetized $\mathrm{C} 57 \mathrm{BL} / 6$ or $\mathrm{C} 3 \mathrm{H}$ mice were infected at day $\mathrm{O}$ by intranasal inoculation of $50 \mu$ l broth containing $M$. pulmonis organisms of strain CT8 (41). The broth contained $10^{6} \mathrm{CFU}$ of passage 4 or $10^{4} \mathrm{CFU}$ of passage 5 M. pulmonis based on virulence. Infection severity was assessed during and at the end of the experiment by survival and change in body weight, lung weight, and bronchial lymph node weight. Mice were studied on day 1 to day 14 after infection. Other C57BL/6 mice were studied 6 or 16 hours after intraperitoneal injection of LPS (15 $\mathrm{mg} / \mathrm{kg}$; Sigma-Aldrich, catalog L2880) or i.v. injection of TNF- $\alpha(0.02$ to $0.24 \mathrm{mg} / \mathrm{kg}$; Peprotech Inc., catalog 315-01).

Treatment with agonists and inhibitors. ANG2 function-blocking human monoclonal antibody (REGN910) (24), Tie2 function-blocking human monoclonal antibody (REGN1376) (76), and recombinant ANG1 and ANG2 (BowANG1 and BowANG2 as Ang- $\mathrm{F}_{1}-\mathrm{Fc}_{\mathrm{C}} \mathrm{F}_{1}$ and Ang$\mathrm{F}_{2}-\mathrm{Fc}-\mathrm{F}_{2}$, denoted $\mathrm{F}$ for fibrinogen-like domain; ref. 21) were injected i.p. REGN910 (5 mg/kg) and REGN1376 (10 mg/kg) were injected twice ( 1 day before and 3 days after inoculation) over the 1-week infection. Control mice received 2 i.p. injections of human IgG $(10 \mathrm{mg} / \mathrm{kg}$, REGN379). BowANG1 and BowANG2 were injected 3 times, 1 day before and days 2 and 5 after infection, at a dose of $25 \mathrm{mg} / \mathrm{kg}$ i.p. The selective pan-class I PI3K inhibitor BKM120 (NVP-BKM120, Novartis) (77), dissolved in 10\% N-methyl-2-pyrrolidone, 90\% PEG 300, was administered by gavage at a dose of $50 \mathrm{mg} / \mathrm{kg} 1$ day before infection and daily thereafter over the 1-week infection. Pathogen-free controls had the same treatment.

Tissue preparation and immunohistochemistry. Tissues were preserved by perfusion of fixative ( $1 \%$ paraformaldehyde in PBS; $\mathrm{pH}$ 7.4) through the left ventricle into the aorta for 2 minutes at a pressure of 120 to $140 \mathrm{mmHg}$. Tracheas were washed and stained as whole mounts for immunohistochemistry using 1 or more antibodies diluted at 1:500 in PBS containing 0.3\% Triton-X 100 and 5\% normal serum, as follows: CD31 (Thermo Scientific, hamster monoclonal, catalog MA3105), ANG2 (Regeneron Pharmaceuticals Inc., human monoclonal, catalog REGN910), GFP (Aves Labs Inc., chicken polyclonal, catalog GFP-1020), vWF (Dako, rabbit polyclonal, catalog A0082), P-selectin (BD Biosciences, rat monoclonal, catalog 550289), golgin-97 (Abcam, rabbit polyclonal, catalog ab84340), Tie1 (R\&D, goat polyclonal, catalog AF619), Tie2 (Regeneron Pharmaceuticals Inc., human monoclonal, catalog REGN1376), phospho-Tie2 (Y992, R\&D, rabbit polyclonal, catalog AF2720), phospho-Tie2 (Y1102/Y1100, R\&D, rabbit polyclonal, catalog AF3909), and FOXO1 (Cell Signaling, rabbit monoclonal, catalog 2880). Species-specific secondary antibodies were labeled with Cy3, Alexa Fluor 488, or Cy5 (1:500; Jackson ImmunoResearch Laboratories Inc.). Specimens were examined with a Zeiss Axiophot fluorescence microscope or Zeiss LSM-510 confocal microscope using AIM 4.0 confocal software.

Microsphere injection. To assess vascular leakage, $50 \mu \mathrm{l}$ of a suspension of 50-nm fluorescent polystyrene microspheres (1\% solids, Bangs Laboratories, Inc.) was injected into the tail vein (36). Mice were anesthetized at 5 minutes and perfused after the microspheres circulated for 10 minutes.

Morphometric measurements. Amounts of immunoreactivity and leakage of fluorescent microspheres were measured as the area density of the fluorescent signal above a predetermined threshold (36) or as the fluorescence intensity in the range of 0 to 255 using Image J software (NIH). See Supplemental Methods for details. Capillary and 
venule diameters were measured in tracheal whole mounts stained for CD31 in endothelial cells. The diameter of 15 to 20 capillaries over 5 cartilage rings and 15 to 20 venules in intercartilaginous regions per trachea was measured at a $\times 10$ objective magnification. Measurements were made on real-time images obtained with the fluorescence microscope $(\times 10$ objective, $\times 1$ Optovar $)$ and Olympus DP73 camera using custom image analysis software. The association of ANG2 with other molecules was assessed by measuring the percentage of voxels that colocalized in individual slices by comparing corresponding channels (colors) of confocal microscopic image stacks with Imaris software (version 8.3, http://www.bitplane.com/imaris).

Statistics. Data are presented as mean \pm SEM with 4 to 21 mice per group. The number of mice in individual experiments is shown in figure legends. Differences were assessed by 2-tailed Student's $t$ test or 1-way ANOVA followed by Bonferroni's test for multiple comparisons. The correlation between ANG2 expression and capillary diameter in pathogen-free Tie1-ANG2 mice was analyzed by linear regression. $P$ values of less than 0.05 were considered statistically significant.

Study approval. All experiments were approved by the Institutional Animal Care and Use Committee of UCSF.

\section{Author contributions}

MK and BA designed and performed experiments, analyzed and interpreted results, and wrote the manuscript. EAK, MN, and HWY contributed to the design of experiments and analysis of data. GT, CD, KA, and PS provided reagents/materials and con- tributed to the design of experiments, data analysis, and interpretation of results. PB provided transgenic mice. DMM contributed to the design of experiments, data analysis, interpretation of results, and writing of the manuscript.

\section{Acknowledgments}

We thank Dan Dumont for Tie1-tTA mice and Stefan Scherer of Novartis for supplying BKM120. This work was supported in part by grants from the National Heart, Lung, and Blood Institute of the NIH (P01 HL024136, R01 HL059157, and R01 HL127402 to DMM), funding from the Leducq Foundation (11CVD03 to KA and DMM), funding from the Angel-Works Foundation (to DMM), and support from the National Research Foundation of Korea funded by the Ministry of Education (2012-R1A6A3A03040326 to MK). Additional support came from the Academy of Finland (Centre of Excellence Program 2014-2019 (271845 to PS and KA), a European Research Council (ERC) Advanced Grant (ERC2010-AdG-268804 to KA), the Marie Curie Actions of European Union Seventh Framework Programme (FP7/2007-2013 grant 317250), the Sigrid Juselius Foundation (to PS and KA), the Finnish Cultural Foundation (to PS), and the Finnish Cancer Organization (to PS, KA, and EAK).

Address correspondence to: Donald M. McDonald, University of California, San Francisco, 513 Parnassus Avenue, Room S1349, San Francisco, California 94143-0452, USA. Phone: 415.476.2118; E-mail: donald.mcdonald@ucsf.edu.
1. Augustin HG, Koh GY, Thurston G, Alitalo K. Control of vascular morphogenesis and homeostasis through the angiopoietin-Tie system. Nat Rev Mol Cell Biol. 2009;10(3):165-177.

2. Baluk $\mathrm{P}$, et al. TNF-alpha drives remodeling of blood vessels and lymphatics in sustained airway inflammation in mice. JClin Invest. 2009;119(10):2954-2964.

3. Lee CG, et al. Vascular endothelial growth factor (VEGF) induces remodeling and enhances TH2-mediated sensitization and inflammation in the lung. Nat Med. 2004;10(10):1095-1103.

4. Tabruyn SP, et al. Angiopoietin-2-driven vascular remodeling in airway inflammation. Am J Pathol. 2010;177(6):3233-3243.

5 . Sato TN, et al. Distinct roles of the receptor tyrosine kinases Tie-1 and Tie-2 in blood vessel formation. Nature. 1995;376(6535):70-74.

6. Suri C, et al. Requisite role of angiopoietin-1, a ligand for the TIE2 receptor, during embryonic angiogenesis. Cell. 1996;87(7):1171-1180.

7. Yancopoulos GD, Davis S, Gale NW, Rudge JS, Wiegand SJ, Holash J. Vascular-specific growth factors and blood vessel formation. Nature. 2000;407(6801):242-248.

8. Puri MC, Rossant J, Alitalo K, Bernstein A, Partanen J. The receptor tyrosine kinase TIE is required for integrity and survival of vascular endothelial cells. EMBO J. 1995;14(23):5884-5891.

9. Saharinen $\mathrm{P}$, et al. Angiopoietins assemble distinct Tie2 signalling complexes in endothelial cell-cell and cell-matrix contacts. Nat Cell Biol. 2008;10(5):527-537.

10. Brunet A, et al. Akt promotes cell survival by phosphorylating and inhibiting a Forkhead transcription factor. Cell. 1999;96(6):857-868.

11. Daly C, et al. Angiopoietin-1 modulates endothelial cell function and gene expression via the transcription factor FKHR (FOXO1). Genes Dev. 2004;18(9):1060-1071.

12. Kim I, Kim HG, So JN, Kim JH, Kwak HJ, Koh GY. Angiopoietin-1 regulates endothelial cell survival through the phosphatidylinositol 3'-Kinase/ Akt signal transduction pathway. Circ Res. 2000;86(1):24-29.

13. Potente $\mathrm{M}$, et al. Involvement of Foxo transcription factors in angiogenesis and postnatal neovascularization. J Clin Invest. 2005;115(9):2382-2392.

14. Zhang X, et al. Phosphorylation of serine 256 suppresses transactivation by FKHR (FOXO1) by multiple mechanisms. Direct and indirect effects on nuclear/cytoplasmic shuttling and DNA binding. J Biol Chem. 2002;277(47):45276-45284.

15. Maisonpierre PC, et al. Angiopoietin-2, a natural antagonist for Tie2 that disrupts in vivo angiogenesis. Science. 1997;277(5322):55-60.

16. Benest AV, et al. Angiopoietin-2 is critical for cytokine-induced vascular leakage. PLOS ONE. 2013;8(8):e70459.

17. Le CT, et al. Synergistic actions of blocking angiopoietin-2 and tumor necrosis factor- $\alpha$ in suppressing remodeling of blood vessels and lymphatics in airway inflammation. Am J Pathol. 2015;185(11):2949-2968.

18. Parikh SM, et al. Excess circulating angiopoietin-2 may contribute to pulmonary vascular leak in sepsis in humans. PLoS Med. 2006;3(3):e46.

19. Ziegler T, et al. Angiopoietin 2 mediates micro- vascular hemodynamic alterations in sepsis. J Clin Invest. 2013;123(8):3436-3445.

20. Daly C, et al. Angiopoietin-2 functions as an autocrine protective factor in stressed endothelial cells. Proc Natl Acad Sci U S A. 2006;103(42):15491-15496.

21. Davis S, et al. Angiopoietins have distinct modular domains essential for receptor binding, dimerization and superclustering. Nat Struct Biol. 2003;10(1):38-44.

22. Scharpfenecker M, Fiedler U, Reiss Y, Augustin HG. The Tie-2 ligand angiopoietin-2 destabilizes quiescent endothelium through an internal autocrine loop mechanism. JCell Sci. 2005;118(Pt 4):771-780.

23. Yuan HT, Khankin EV, Karumanchi SA, Parikh SM. Angiopoietin 2 is a partial agonist/antagonist of Tie2 signaling in the endothelium. Mol Cell Biol. 2009;29(8):2011-2022.

24. Daly C, et al. Angiopoietin-2 functions as a Tie2 agonist in tumor models, where it limits the effects of VEGF inhibition. Cancer Res. 2013;73(1):108-118.

25. Hammes HP, et al. Angiopoietin-2 causes pericyte dropout in the normal retina: evidence for involvement in diabetic retinopathy. Diabetes. 2004;53(4):1104-1110.

26. Holash J, et al. Vessel cooption, regression, and growth in tumors mediated by angiopoietins and VEGF. Science. 1999;284(5422):1994-1998.

27. Fiedler U, et al. Angiopoietin-2 sensitizes endothelial cells to TNF-alpha and has a crucial role in the induction of inflammation. Nat Med. 2006;12(2):235-239.

28. Fiedler U, et al. The Tie-2 ligand angiopoietin-2 is 
stored in and rapidly released upon stimulation from endothelial cell Weibel-Palade bodies. Blood. 2004;103(11):4150-4156.

29. Singh H, Hansen TM, Patel N, Brindle NP. The molecular balance between receptor tyrosine kinases Tie 1 and Tie 2 is dynamically controlled by VEGF and TNF $\alpha$ and regulates angiopoietin signalling. PLoS ONE. 2012;7(1):e29319.

30. Bureau W, et al. Chronic systemic delivery of angiopoietin-2 reveals a possible independent angiogenic effect. Am J Physiol Heart Circ Physiol. 2006;291(2):H948-H956.

31. Kim KE, Cho CH, Kim HZ, Baluk P, McDonald DM, Koh GY. In vivo actions of angiopoietins on quiescent and remodeling blood and lymphatic vessels in mouse airways and skin. Arterioscler Thromb Vasc Biol. 2007;27(3):564-570.

32. Thurston G, et al. Leakage-resistant blood vessels in mice transgenically overexpressing angiopoietin-1. Science. 1999;286(5449):2511-2514.

33. Boscolo E, et al. Rapamycin improves TIE2-mutated venous malformation in murine model and human subjects. JClin Invest. 2015;125(9):3491-3504.

34. Vikkula M, et al. Vascular dysmorphogenesis caused by an activating mutation in the receptor tyrosine kinase TIE2. Cell. 1996;87(7):1181-1190.

35. Fuxe J, et al. Angiopoietin/Tie2 signaling transforms capillaries into venules primed for leukocyte trafficking in airway inflammation. Am J Pathol. 2010;176(4):2009-2018.

36. Fuxe J, et al. Pericyte requirement for anti-leak action of angiopoietin- 1 and vascular remodeling in sustained inflammation. Am J Pathol. 2011;178(6):2897-2909.

37. Thurston G, et al. Angiopoietin-1 protects the adult vasculature against plasma leakage. $\mathrm{Nat}$ Med. 2000;6(4):460-463.

38. Korhonen EA, et al. Tie1 controls angiopoietin function in vascular remodeling and inflammation. J Clin Invest. 2016;126(9):3495-3510.

39. Sarao R, Dumont DJ. Conditional transgene expression in endothelial cells. Transgenic Res. 1998;7(6):421-427.

40. Baluk P, et al. Regulated angiogenesis and vascular regression in mice overexpressing vascular endothelial growth factor in airways. Am J Pathol. 2004;165(4):1071-1085.

41. Thurston G, Murphy TJ, Baluk P, Lindsey JR, McDonald DM. Angiogenesis in mice with chronic airway inflammation: strain-dependent differences. Am J Pathol. 1998;153(4):1099-1112.

42. Ezaki T, Baluk P, Thurston G, La Barbara A, Woo $\mathrm{C}, \mathrm{McD}$ onald DM. Time course of endothelial cell proliferation and microvascular remodeling in chronic inflammation. Am J Pathol. 2001;158(6):2043-2055.

43. Gong S, et al. A gene expression atlas of the central nervous system based on bacterial artificial chromosomes. Nature. 2003;425(6961):917-925.

44. Cartner SC, Simecka JW, Lindsey JR, Cassell GH, Davis JK. Chronic respiratory mycoplasmosis in $\mathrm{C} 3 \mathrm{H} / \mathrm{HeN}$ and $\mathrm{C} 57 \mathrm{BL} / 6 \mathrm{~N}$ mice: lesion severity and antibody response. Infect Immun. 1995;63(10):4138-4142.
45. Wong AL, Haroon ZA, Werner S, Dewhirst MW, Greenberg CS, Peters KG. Tie2 expression and phosphorylation in angiogenic and quiescent adult tissues. Circ Res. 1997;81(4):567-574.

46. Jones N, et al. Identification of Tek/Tie2 binding partners. Binding to a multifunctional docking site mediates cell survival and migration. J Biol Chem. 1999;274(43):30896-30905.

47. Kontos CD, et al. Tyrosine 1101 of Tie 2 is the major site of association of $\mathrm{p} 85$ and is required for activation of phosphatidylinositol 3-kinase and Akt. Mol Cell Biol. 1998;18(7):4131-4140.

48. Suri $\mathrm{C}$, et al. Increased vascularization in mice overexpressing angiopoietin-1. Science. 1998;282(5388):468-471.

49. Baffert F, Thurston G, Rochon-Duck M, Le T, Brekken R, McDonald DM. Age-related changes in vascular endothelial growth factor dependency and angiopoietin-1-induced plasticity of adult blood vessels. Circ Res. 2004;94(7):984-992.

50. Cho $\mathrm{CH}$, et al. Long-term and sustained COMP-Ang1 induces long-lasting vascular enlargement and enhanced blood flow. Circ Res. 2005;97(1):86-94.

51. Hackett SF, Wiegand S, Yancopoulos G, Campochiaro PA. Angiopoietin-2 plays an important role in retinal angiogenesis. J Cell Physiol. 2002;192(2):182-187.

52. Reiss $\mathrm{Y}$, et al. Angiopoietin-2 impairs revascularization after limb ischemia. Circ Res. 2007;101(1):88-96.

53. Yabkowitz R, Meyer S, Black T, Elliott G, Merewether LA, Yamane HK. Inflammatory cytokines and vascular endothelial growth factor stimulate the release of soluble tie receptor from human endothelial cells via metalloprotease activation. Blood.1999;93(6):1969-1979.

54. Cho $\mathrm{CH}$, et al. COMP-Ang1: a designed angiopoietin-1 variant with nonleaky angiogenic activity. Proc Natl Acad Sci U S A. 2004;101(15):5547-5552.

55. McDonald DM. Endothelial gaps and permeability of venules in rat tracheas exposed to inflammatory stimuli. Am J Physiol. 1994;266(1 Pt 1):L61-L83.

56. Gale NW, et al. Complementary and coordinated roles of the VEGFs and angiopoietins during normal and pathologic vascular formation. Cold Spring Harb Symp Quant Biol. 2002;67:267-273.

57. Bogdanovic E, Nguyen VP, Dumont DJ. Activation of Tie 2 by angiopoietin-1 and angiopoietin-2 results in their release and receptor internalization. JCell Sci. 2006;119(Pt 17):3551-3560.

58. Kim I, Kim JH, Moon SO, Kwak HJ, Kim NG, Koh GY. Angiopoietin-2 at high concentration can enhance endothelial cell survival through the phosphatidylinositol 3'-kinase/ Akt signal transduction pathway. Oncogene. 2000;19(39):4549-4552.

59. Teichert-Kuliszewska K, et al. Biological action of angiopoietin-2 in a fibrin matrix model of angiogenesis is associated with activation of Tie2. Cardiovasc Res. 2001;49(3):659-670.

60. Shen J, et al. Targeting VE-PTP activates TIE2 and stabilizes the ocular vasculature. JClin Invest. 2014;124(10):4564-4576.
61. Gale NW, et al. Angiopoietin-2 is required for postnatal angiogenesis and lymphatic patterning, and only the latter role is rescued by Angiopoietin-1. Dev Cell. 2002;3(3):411-423.

62. Pitera JE, Woolf AS, Gale NW, Yancopoulos GD, Yuan HT. Dysmorphogenesis of kidney cortical peritubular capillaries in angiopoietin-2-deficient mice. Am J Pathol. 2004;165(6):1895-1906.

63. Shimoda H, Bernas MJ, Witte MH, Gale NW, Yancopoulos GD, Kato S. Abnormal recruitment of periendothelial cells to lymphatic capillaries in digestive organs of angiopoietin-2-deficient mice. Cell Tissue Res. 2007;328(2):329-337.

64. Dellinger $\mathrm{M}$, et al. Defective remodeling and maturation of the lymphatic vasculature in Angiopoietin-2 deficient mice. Dev Biol. 2008;319(2):309-320.

65. Brown JL, et al. A human monoclonal anti-ANG2 antibody leads to broad antitumor activity in combination with VEGF inhibitors and chemotherapy agents in preclinical models. Mol Cancer Ther. 2010;9(1):145-156.

66. Felcht M, et al. Angiopoietin-2 differentially regulates angiogenesis through TIE2 and integrin signaling. J Clin Invest. 2012;122(6):1991-2005.

67. Hakanpaa L, et al. Endothelial destabilization by angiopoietin-2 via integrin $\beta 1$ activation. $N a t$ Commun. 2015;6:5962.

68. Furuyama T, et al. Abnormal angiogenesis in Foxo1 (Fkhr)-deficient mice. J Biol Chem. 2004;279(33):34741-34749.

69. Taddei A, et al. Endothelial adherens junctions control tight junctions by VE-cadherin-mediated upregulation of claudin-5. Nat Cell Biol. 2008;10(8):923-934.

70. Choi HJ, et al. Yes-associated protein regulates endothelial cell contact-mediated expression of angiopoietin-2. Nat Commun. 2015;6:6943.

71. McClatchey AI, Yap AS. Contact inhibition (of proliferation) redux. Curr Opin Cell Biol. 2012;24(5):685-694.

72. Papapetropoulos A, et al. Angiopoietin-1 inhibits endothelial cell apoptosis via the Akt/survivin pathway. J Biol Chem. 2000;275(13):9102-9105.

73. Kanda S, Miyata Y, Mochizuki Y, Matsuyama $\mathrm{T}$, Kanetake H. Angiopoietin 1 is mitogenic for cultured endothelial cells. Cancer Res. 2005;65(15):6820-6827.

74. Fukuhara S, et al. Differential function of Tie2 at cell-cell contacts and cell-substratum contacts regulated by angiopoietin-1. Nat Cell Biol. 2008;10(5):513-526.

75. Lee J, et al. Angiopoietin-1 guides directional angiogenesis through integrin $\alpha v \beta 5$ signaling for recovery of ischemic retinopathy. Sci Transl Med. 2013;5(203):203ra127.

76. Adler AP, et al. Blockade of angiopoietin-2 or Tie2 is equally effective at inhibiting tumor growth and reducing tumor vessel density in most human tumor xenograft models [AACR abstract 4492]. Cancer Res. 2014;74(19 suppl).

77. Lonetti A, et al. Activity of the pan-class I phosphoinositide 3-kinase inhibitor NVP-BKM120 in T-cell acute lymphoblastic leukemia. Leukemia. 2014;28(6):1196-1206. 